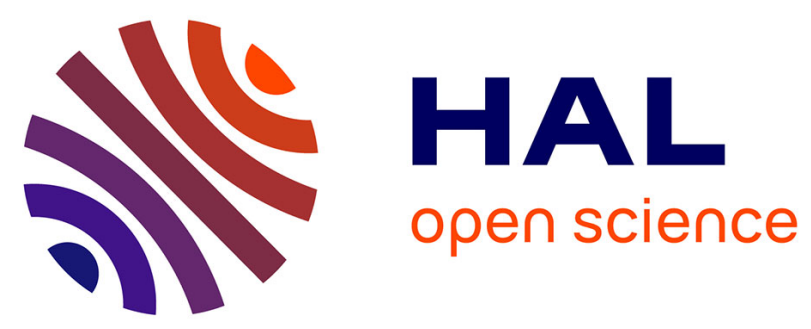

\title{
A DENSITY RESULT FOR GSBD AND ITS APPLICATION TO THE APPROXIMATION OF BRITTLE FRACTURE ENERGIES
}

Flaviana Iurlano

\section{- To cite this version:}

Flaviana Iurlano. A DENSITY RESULT FOR GSBD AND ITS APPLICATION TO THE APPROXIMATION OF BRITTLE FRACTURE ENERGIES. Calculus of Variations and Partial Differential Equations, 2014, 51 (1-2), pp.315-342. 10.1007/s00526-013-0676-7 . hal-02145304

\section{HAL Id: hal-02145304 \\ https://hal.science/hal-02145304}

Submitted on 6 Jun 2019

HAL is a multi-disciplinary open access archive for the deposit and dissemination of scientific research documents, whether they are published or not. The documents may come from teaching and research institutions in France or abroad, or from public or private research centers.
L'archive ouverte pluridisciplinaire HAL, est destinée au dépôt et à la diffusion de documents scientifiques de niveau recherche, publiés ou non, émanant des établissements d'enseignement et de recherche français ou étrangers, des laboratoires publics ou privés. 


\title{
A DENSITY RESULT FOR GSBD AND ITS APPLICATION TO THE APPROXIMATION OF BRITTLE FRACTURE ENERGIES
}

\author{
FLAVIANA IURLANO
}

\begin{abstract}
We present an approximation result for functions $u: \Omega \rightarrow \mathbb{R}^{n}$ belonging to the space $\operatorname{GSBD}(\Omega) \cap L^{2}\left(\Omega, \mathbb{R}^{n}\right)$ with $e(u)$ square integrable and $\mathcal{H}^{n-1}\left(J_{u}\right)$ finite. The approximating functions $u_{k}$ are piecewise continuous functions such that $u_{k} \rightarrow u$ in $L^{2}\left(\Omega, \mathbb{R}^{n}\right), e\left(u_{k}\right) \rightarrow e(u)$ in $L^{2}\left(\Omega, \mathbb{M}_{s y m}^{n \times n}\right), \mathcal{H}^{n-1}\left(J_{u_{k}} \triangle J_{u}\right) \rightarrow 0$, and $\int_{J_{u_{k}} \cup J_{u}}\left|u_{k}^{ \pm}-u^{ \pm}\right| \wedge 1 d \mathcal{H}^{n-1} \rightarrow 0$. As an application, we provide the extension to the vector-valued case of the $\Gamma$-convergence result in $S B V(\Omega)$ proved by Ambrosio and Tortorelli in $[4,5]$.
\end{abstract}

\section{INTRODUCTION}

A typical example of the minimum problems occurring in the mathematical formulation of some variational models in Linearly Elastic Fracture Mechanics (see, e.g., [20, 21], [10]) is

$$
\min _{u}\left(\int_{\Omega \backslash J_{u}} Q(e(u)) d x+\mathcal{H}^{n-1}\left(J_{u}\right)+\int_{\Omega}|u-g|^{2} d x\right)
$$

where $\Omega \subset \mathbb{R}^{n}$ is a bounded open set, $Q$ is a positive definite quadratic form on the space of symmetric $n \times n$ matrices, $\mathcal{H}^{n-1}$ is the $(n-1)$-Hausdorff measure in $\mathbb{R}^{n}, g \in L^{2}\left(\Omega, \mathbb{R}^{n}\right), e(u)$ is the symmetric part of the gradient of $u$, and $J_{u}$ is the jump set of $u$.

For a numerical treatment of these minimum problems, a standard approach is to approximate (1.1) with functionals defined on a class of functions without jumps. Drawing inspiration from the scalar-valued case, numerical computations concerning (1.1) and similar problems are performed, e.g., in $[9,10]$, and $[8]$ using a phase-field approximation, which leads to the minimization of Ambrosio-Tortorelli type functionals $[4,5]$

$$
\min _{(u, v)} \int_{\Omega}\left(v Q(e(u))+\frac{(1-v)^{2}}{\varepsilon_{k}}+\varepsilon_{k}|\nabla v|^{2}+|u-g|^{2}\right) d x,
$$

where $\eta_{k}, \varepsilon_{k}$ belong to $(0,+\infty), \eta_{k} / \varepsilon_{k} \rightarrow 0$, and $(u, v)$ runs in $H^{1}\left(\Omega, \mathbb{R}^{n}\right) \times H^{1}(\Omega)$ with $\eta_{k} \leq v \leq 1$.

Nevertheless, the rigorous convergence of these minimum problems to the problem (1.1) has not yet been proved in the vector-valued case. An important contribution in this direction has been given by Chambolle in [12,13], where the problem (1.1) is set in the space $S B D(\Omega)$ (we refer to [23] for its definition) and the convergence result is proved under the assumption of an a priori bound on the $L^{\infty}$-norm of the functions $u$. Actually, even the existence of solutions in $S B D(\Omega)$ to the problem (1.1) is guaranteed only if an a priori $L^{\infty}$-bound for minimizing sequences is assumed (see [7, Theorem 3.1]).

In the present paper we provide the first complete proof of the convergence of the solutions to (1.2) toward a solution to (1.1), formulating these problems in a more convenient framework. Precisely, if $\left(u_{k}, v_{k}\right)$ is a sequence of minimizers of the problem (1.2), we prove (see Corollary 4.2) that $v_{k} \rightarrow 1$ in $L^{1}(\Omega)$ and a subsequence of $u_{k}$ converges in $L^{2}\left(\Omega, \mathbb{R}^{n}\right)$ to a minimizer $u$ of the problem (1.1) in the space $G S B D(\Omega)$ of Generalized Special Functions of Bounded Deformation.

2010 Mathematics Subject Classification. Primary: 49Q20, 49J45; Secondary: 26B30, 74R10, 35A35.

Key words and phrases. Generalized functions of bounded deformation, free discontinuity problems, brittle fracture.

Preprint SISSA 38/2012/M. 
This space has been recently introduced by Dal Maso in [17] to solve minimum problems of the form (1.1) without $L^{\infty}$-bounds on the minimizing sequences. For every $u \in G S B D(\Omega)$ one can define the approximate one-sided limits $u^{ \pm}$on regular submanifolds, the approximate jump set $J_{u}$, which turns out to be $\left(\mathcal{H}^{n-1}, n-1\right)$-rectifiable, and the approximate symmetric gradient $e(u) \in L^{1}\left(\Omega, \mathbb{M}_{\text {sym }}^{n \times n}\right)$ (see Section 2 for a summary of these fine properties of $G S B D$-functions). Therefore the functional occurring in (1.1) makes sense in this more general context and a solution in $G S B D(\Omega)$ to the minimum problem is ensured by the compactness and semicontinuity result proved in [17, Theorem 11.3].

The proof of the convergence of (1.2) to (1.1) is obtained in three steps, following the approach in $[12,13]$. The first (and crucial) step allows us (see Density Theorem 3.1) to approximate a function $u \in G S B D(\Omega) \cap L^{2}\left(\Omega, \mathbb{R}^{n}\right)$, for which $e(u)$ is square integrable and $\mathcal{H}^{n-1}\left(J_{u}\right)$ is finite, with a sequence $\left(u_{k}\right) \subset S B V\left(\Omega, \mathbb{R}^{n}\right) \cap L^{\infty}\left(\Omega, \mathbb{R}^{n}\right)$ of piecewise continuous functions in a way that

$$
\begin{gathered}
\left\|u_{k}-u\right\|_{L^{2}\left(\Omega, \mathbb{R}^{n}\right)} \rightarrow 0, \\
\left\|e\left(u_{k}\right)-e(u)\right\|_{L^{2}\left(\Omega, \mathbb{M}_{s y m}^{n \times n}\right)} \rightarrow 0, \\
\mathcal{H}^{n-1}\left(J_{u_{k}} \triangle J_{u}\right) \rightarrow 0, \\
\int_{J_{u_{k}} \cup J_{u}}\left|u_{k}^{ \pm}-u^{ \pm}\right| \wedge 1 d \mathcal{H}^{n-1} \rightarrow 0,
\end{gathered}
$$

where $\triangle$ denotes the symmetric difference and $a \wedge b:=\min \{a, b\}$.

The second step concerns the $\Gamma$-convergence of the functionals occurring in (1.2) to the one occurring in (1.1) (see Theorem 4.1). In particular the Density Theorem 3.1 is involved in the proof of the $\Gamma$-lim sup inequality, allowing us to construct a recovery sequence starting from more regular functions.

The third step is the proof of the compactness of the minimizing sequences of (1.2). This is obtained in Proposition 4.5 using a characterization which relates $L^{1}$-compactness of sequences with $L^{1}$-compactness of slices (see [1, Theorem 6.6] and [17, Lemma 10.7]). The convergence of minima and minimizers eventually follows from well-known results in $\Gamma$-convergence theory.

The paper is organized as follows. In Section 2 we supply the essential notation and preliminaries. Sections 3 is devoted to state and prove the Density Theorem 3.1. Finally in Section 4 we show the application of the density theorem to the Ambrosio-Tortorelli approximation of (1.1).

\section{Notation And PREliminaries}

Let $n \geq 2$ be a fixed integer. The Lebesgue measure and the $k$-dimensional Hausdorff measure in $\mathbb{R}^{n}$ are denoted by $\mathcal{L}^{n}$ and $\mathcal{H}^{k}$, respectively. For every set $A$ the characteristic function $\chi_{A}$ is defined by $\chi_{A}(x):=1$ if $x \in A$ and by $\chi_{A}(x):=0$ if $x \notin A$. Throughout the paper $\Omega$ is assumed to be a bounded open subset of $\mathbb{R}^{n}$. Moreover $c$ will denote a constant which may vary from line to line.

BV-functions. For the definitions and the main properties of $B V\left(\Omega, \mathbb{R}^{n}\right)$, of $S B V\left(\Omega, \mathbb{R}^{n}\right)$, of the distributional derivative $D u$ of a function $u \in B V\left(\Omega, \mathbb{R}^{n}\right)$, of the approximate gradient $\nabla u$, of the approximate one-sided limits $u^{ \pm}$on regular submanifolds, of the jump function $[u]:=u^{+}-u^{-}$, and of the approximate jump set $J_{u}$ we refer to [3]. Here we only recall the definition of the space $S B V^{p}\left(\Omega, \mathbb{R}^{n}\right)$, with $1<p<+\infty$, used in the sequel:

$$
S B V^{p}\left(\Omega, \mathbb{R}^{n}\right):=\left\{u \in S B V\left(\Omega, \mathbb{R}^{n}\right): \nabla u \in L^{p}\left(\Omega, \mathbb{M}^{n \times n}\right) \text { and } \mathcal{H}^{n-1}\left(J_{u}\right)<+\infty\right\},
$$

being $\mathbb{M}^{n \times n}$ the space of all $n \times n$ matrices.

BD-functions. For the definitions and the main properties of $B D(\Omega)$, of $S B D(\Omega)$, of the symmetric distributional derivative $E u$ of a function $u \in B D(\Omega)$, of the approximate symmetric gradient $e(u)$, of the approximate one-sided limits $u^{ \pm}$on regular submanifolds, of the jump function $[u]:=u^{+}-u^{-}$, of the approximate jump set $J_{u}$ we refer to [24], [23], [6], and [2]. 
We point out that if $\Omega$ has Lipschitz boundary and $u \in L^{1}\left(\Omega, \mathbb{R}^{n}\right)$ satisfies $E u \in L^{2}\left(\Omega, \mathbb{M}_{\text {sym }}^{n \times n}\right)$, where $\mathbb{M}_{\text {sym }}^{n \times n}$ is the set of all $n \times n$ symmetric matrices, then $u$ actually belongs to $H^{1}\left(\Omega, \mathbb{R}^{n}\right)$. This can be obtained arguing as in the proof of [23, Proposition 1.1].

We define $S B D^{p}(\Omega), 1<p<+\infty$, by

$$
S B D^{p}(\Omega):=\left\{u \in S B D\left(\Omega, \mathbb{R}^{n}\right): e(u) \in L^{p}\left(\Omega, \mathbb{M}_{\text {sym }}^{n \times n}\right) \text { and } \mathcal{H}^{n-1}\left(J_{u}\right)<+\infty\right\} .
$$

Slices. Fixed $\xi \in \mathbb{S}^{n-1}:=\left\{\xi \in \mathbb{R}^{n}:|\xi|=1\right\}$, we set

$$
\Pi^{\xi}:=\left\{y \in \mathbb{R}^{n}: y \cdot \xi=0\right\}, \quad \Omega_{y}^{\xi}:=\{t \in \mathbb{R}: y+t \xi \in \Omega\} \text { for } y \in \Pi^{\xi}, \quad \Omega^{\xi}:=\left\{y \in \Pi^{\xi}: \Omega_{y}^{\xi} \neq \varnothing\right\} .
$$

For $u: \Omega \rightarrow \mathbb{R}^{n}$ and for $e: \Omega \rightarrow \mathbb{M}_{\text {sym }}^{n \times n}$ we define the slices $u_{y}^{\xi}, e_{y}^{\xi}: \Omega_{y}^{\xi} \rightarrow \mathbb{R}$ by

$$
u_{y}^{\xi}(t):=u(y+t \xi) \cdot \xi \quad \text { and } \quad e_{y}^{\xi}(t):=e(y+t \xi) \xi \cdot \xi .
$$

If $u_{k}, u \in L^{1}\left(\Omega, \mathbb{R}^{n}\right)$ and $u_{k} \rightarrow u$ in $L^{1}\left(\Omega, \mathbb{R}^{n}\right)$, then for every $\xi \in S^{n-1}$ there exists a subsequence $\left(u_{k_{j}}\right)$ such that

$$
\left(u_{k_{j}}\right)_{y}^{\xi} \rightarrow u_{y}^{\xi} \text { in } L^{1}\left(\Omega_{y}^{\xi}\right) \text { for } \mathcal{H}^{n-1} \text {-a.e. } y \in \Omega^{\xi} .
$$

GBD-functions. We now summarize the definition and the main properties of $G B D$-functions, referring to [17] for more details.

Let us denote by $\mathcal{M}_{b}(\Omega)$ the set of all bounded Radon measures in $\Omega$ and by $\mathcal{M}_{b}^{+}(\Omega)$ the set of nonnegative ones.

Definition 2.1. An $\mathcal{L}^{n}$-measurable function $u: \Omega \rightarrow \mathbb{R}^{n}$ belongs to $G B D(\Omega)$ if there exists $\lambda_{u} \in$ $\mathcal{M}_{b}^{+}(\Omega)$ such that the following equivalent condition hold for every $\xi \in \mathbb{S}^{n-1}$ :

(a) for every $\tau \in C^{1}(\mathbb{R})$ with $-\frac{1}{2} \leq \tau \leq \frac{1}{2}$ and $0 \leq \tau^{\prime} \leq 1$, the partial derivative $D_{\xi}(\tau(u \cdot \xi))$ belongs to $\mathcal{M}_{b}(\Omega)$ and its total variation satisfies

$$
\left|D_{\xi}(\tau(u \cdot \xi))\right|(B) \leq \lambda_{u}(B),
$$

for every Borel set $B \subset \Omega$;

(b) for $\mathcal{H}^{n-1}$-a.e. $y \in \Omega^{\xi}$ the function $u_{y}^{\xi}$ belongs to $B V_{\text {loc }}\left(\Omega_{y}^{\xi}\right)$ and for every Borel set $B \subset \Omega$ it satisfies

$$
\int_{\Omega^{\xi}}\left(\left|D u_{y}^{\xi}\right|\left(B_{y}^{\xi} \backslash J_{u_{y}^{\xi}}^{1}\right)+\mathcal{H}^{0}\left(B_{y}^{\xi} \cap J_{u_{y}^{\xi}}^{1}\right)\right) d \mathcal{H}^{n-1} \leq \lambda_{u}(B),
$$

where we have set

$$
J_{u_{y}^{\xi}}^{1}:=\left\{t \in J_{u_{y}^{\xi}}:\left|\left[u_{y}^{\xi}\right](t)\right| \geq 1\right\} .
$$

The space $G S B D(\Omega)$ is the set of all functions $u \in G B D(\Omega)$ such that for every $\xi \in \mathbb{S}^{n-1}$ and for $\mathcal{H}^{n-1}$-a.e. $y \in \Omega^{\xi}$ the function $u_{y}^{\xi}$ belongs to $S B V_{\text {loc }}\left(\Omega_{y}^{\xi}\right)$.

For every $u \in G B D(\Omega)$ one can define the approximate one-sided limits $u^{ \pm}$on regular submanifolds, the jump function $[u]:=u^{+}-u^{-}$, and the approximate jump set $J_{u}$, which turns out to be $\left(\mathcal{H}^{n-1}, n-1\right)$-rectifiable $[17$, Sections 5 and 6$]$.

Let $\xi \in \mathbb{S}^{n-1}$ and let

$$
J_{u}^{\xi}:=\left\{x \in J_{u}: u^{+}(x) \cdot \xi-u^{-}(x) \cdot \xi \neq 0\right\} .
$$

Then for $\mathcal{H}^{n-1}$-a.e. $y \in \Omega^{\xi}$ we have

$$
\begin{gathered}
\left(J_{u}^{\xi}\right)_{y}^{\xi}=J_{u_{y}^{\xi}}, \\
u^{ \pm}(y+t \xi) \cdot \xi=\left(u_{y}^{\xi}\right)^{ \pm}(t) \quad \text { for every } t \in\left(J_{u}\right)_{y}^{\xi},
\end{gathered}
$$

where the normals to $J_{u}$ and $J_{u_{y}^{\xi}}$ are oriented so that $\xi \cdot \nu_{u} \geq 0$ and $\nu_{u_{y}^{\xi}}=1$.

For $u \in G B D(\Omega)$ the approximate symmetric gradient $e(u)$ in the sense of [6, Definition 8.1] exists and belongs to $L^{1}\left(\Omega ; \mathbb{M}_{\text {sym }}^{n \times n}\right)$. Moreover for every $\xi \in \mathbb{R}^{n} \backslash\{0\}$ and for $\mathcal{H}^{n-1}$-a.e. $y \in \Omega^{\xi}$ one has

$$
(e(u))_{y}^{\xi}=\nabla u_{y}^{\xi} \mathcal{L}^{1} \text {-a.e. on } \Omega_{y}^{\xi} .
$$

Let us define $G S B D^{p}(\Omega)$ for $1<p<+\infty$ as in $(2.1)$, with $S B D(\Omega)$ replaced by $G S B D(\Omega)$. 
Using the Fubini Theorem one can shows that

$$
\mathcal{H}^{n-1}\left(J_{u} \backslash J_{u}^{\xi}\right)=0
$$

for $\mathcal{H}^{n-1}$-a.e. $\xi \in \mathbb{S}^{n-1}$. With the following lemma we deduce the existence of an orthonormal basis $\left(e_{i}\right)_{i=1}^{n}$ for which (2.9) holds for every $\xi \in D:=\left\{e_{i}\right.$ for $i=1, \ldots, n, e_{i} \pm e_{j}$ for $\left.1 \leq i<j \leq n\right\}$.

We denote by $\mu$ the invariant Radon measure on the rotation group $S O(n)$ with $\mu(S O(n))=$ $\mathcal{H}^{n-1}\left(\mathbb{S}^{n-1}\right)$.

Lemma 2.2. Let $\xi_{1}, \ldots, \xi_{k} \in \mathbb{S}^{n-1}$. Then each $\xi \in\left\{R \xi_{1}, \ldots, R \xi_{k}\right\}$ satisfies (2.9), for $\mu$-a.e. $R \in S O(n)$.

Proof. Let $N \subset \mathbb{S}^{n-1}$ be the set where (2.9) fails and let

$$
M_{j}:=\left\{R \in S O(n): R \xi_{j} \in N\right\} .
$$

For $j=1, \ldots, k$ we have

$$
\mu\left(M_{j}\right)=\mathcal{H}^{n-1}(N)=0 .
$$

Therefore for every $R \notin \bigcup_{j=1}^{k} M_{j}$ we find that $R \xi_{1}, \ldots, R \xi_{k} \notin N$ and this concludes the proof.

The following remark is about the extension by zero of $G B D$-functions.

Remark 2.3. Assume that $\Omega$ has Lipschitz boundary and consider a bounded open set $\hat{\Omega}$ with $\Omega \subset \hat{\Omega}$. Let $u \in G B D(\Omega) \cap L^{1}\left(\Omega, \mathbb{R}^{n}\right)$ and let us define $\hat{u}: \hat{\Omega} \rightarrow \mathbb{R}^{n}$ by $\hat{u}:=u$ in $\Omega$ and by $\hat{u}:=0$ outside of $\Omega$. Then the extension $\hat{u}$ belongs to $G B D(\hat{\Omega})$. Indeed, for every $\xi \in \mathbb{S}^{n-1}$ and for $\mathcal{H}^{n-1}$-a.e. $y \in \Omega^{\xi}$ the slice $u_{y}^{\xi}$ belongs to $B V\left(\Omega_{y}^{\xi}\right)$. Since $\Omega$ has Lipschitz boundary, for every $\xi \in \mathbb{S}^{n-1}$ and for $\mathcal{H}^{n-1}$-a.e. $y \in \Omega^{\xi}$ the set $\Omega_{y}^{\xi}$ has finitely many connected components, so that $\hat{u}_{y}^{\xi} \in B V(\mathbb{R})$. Moreover an easy computation and the coarea formula show that

$$
\int_{\hat{\Omega}^{\xi}}\left(\left|D \hat{u}_{y}^{\xi}\right|\left(B_{y}^{\xi} \backslash J_{\hat{u}_{y}^{\xi}}^{1}\right)+\mathcal{H}^{0}\left(B_{y}^{\xi} \cap J_{\hat{u}_{y}^{\xi}}^{1}\right)\right) d \mathcal{H}^{n-1} \leq \lambda_{u}(B \cap \Omega)+\mathcal{H}^{n-1}\lfloor\partial \Omega(B),
$$

for every Borel set $B \subset \hat{\Omega}$ and for $\lambda_{u}$ satisfying (2.4).

The next result provides an estimate for the trace $\operatorname{tr}(u)$ of a function $u \in G S B D(\Omega) \cap L^{1}\left(\Omega, \mathbb{R}^{n}\right)$ (for the definition of $\operatorname{tr}(u)$ see [17, Section 5]).

Lemma 2.4. Assume that $\Omega$ has Lipschitz boundary and define $\tau(s):=\frac{1}{\pi} \operatorname{arctg}(s)$ for $s \in \mathbb{R}$. Then there exists a constant $c(\Omega)<+\infty$, depending on $\Omega$, such that

$$
\int_{\partial \Omega} \tau(|\operatorname{tr}(u)|) d \mathcal{H}^{n-1} \leq c(\Omega)\left(\|u\|_{L^{1}\left(\Omega, \mathbb{R}^{n}\right)}+\lambda_{u}(\Omega)\right)
$$

holds for every $u \in G S B D(\Omega) \cap L^{1}\left(\Omega, \mathbb{R}^{n}\right)$ and for $\lambda_{u} \in \mathcal{M}_{b}^{+}(\Omega)$ satisfying (2.4).

Proof. It is not restrictive to assume that $\Omega$ has the form

$$
\left\{y+t \eta \in \mathbb{R}^{n}: y \in B^{\eta}, 0<t<a(y)\right\}
$$

and that $u$ has compact support in $\Omega \cup \operatorname{graph}(a)$, where $\eta \in \mathbb{S}^{n-1}, B^{\eta} \subset \Pi^{\eta}$ is a relatively open ball, and $a: \overline{B^{\eta}} \rightarrow \mathbb{R}$ is a Lipschitz function. Indeed, let $\left(A_{i}\right)_{i=1}^{k}$ be an open covering of $\partial \Omega$ in a way that $A_{i} \cap \Omega$ has the form (2.11). Let $A_{0} \subset \subset \Omega$ be such that $\left(A_{i}\right)_{i=0}^{k}$ covers $\bar{\Omega}$. Let us consider also a partition of unity $\left(\varphi_{i}\right)_{i=0}^{k}$, such that $\varphi_{i} \in C_{c}^{\infty}\left(A_{i}\right), 0 \leq \varphi_{i} \leq 1$, and $\sum_{i=0}^{k} \varphi_{i}=1$ on $\bar{\Omega}$. Then each $\varphi_{i} u$ belongs to $G S B D\left(A_{i} \cap \Omega\right) \cap L^{1}\left(A_{i} \cap \Omega, \mathbb{R}^{n}\right)$ and has compact support in $A_{i} \cap \bar{\Omega}$. Moreover $\varphi_{i} u$ satisfies (2.4) with $\lambda_{u}(B)$ replaced by

$$
\left\|\nabla \varphi_{i}\right\|_{L^{\infty}\left(A_{i}\right)} \int_{B}|u| d x+\lambda_{u}(B)
$$

for every Borel set $B \subset A_{i} \cap \Omega$. Note that the measure defined in (2.12) belongs to $\mathcal{M}_{b}^{+}\left(A_{i} \cap \Omega\right)$. 
Using the triangle inequality for $\tau$ and inequality (2.10) for $\varphi_{i} u$ with the measure (2.12), we obtain

$$
\begin{aligned}
\int_{\partial \Omega} \tau(|\operatorname{tr}(u)|) d \mathcal{H}^{n-1} & \leq \sum_{i=1}^{k} \int_{A_{i} \cap \partial \Omega} \tau\left(\left|\operatorname{tr}\left(\varphi_{i} u\right)\right|\right) d \mathcal{H}^{n-1} \\
& \leq c\left(\|u\|_{L^{1}\left(\Omega, \mathbb{R}^{n}\right)}+\lambda_{u}(\Omega)\right),
\end{aligned}
$$

where $c<+\infty$ depends on $\Omega$ and $\left(\varphi_{i}\right)_{i=1}^{k}$.

Let us prove now (2.10) under the assumption that $\Omega$ has the form (2.11) and that $u$ has compact support on $\Omega \cup \operatorname{graph}(a)$. We may also assume that there exists a basis $\left(\eta_{i}\right)_{i=1}^{n}$ such that $M:=$ $\operatorname{graph}(\mathrm{a})$ is still a Lipschitz graph in the direction determined by each $\eta_{i}$ and that $\nu(x) \cdot \eta_{i}>\delta>0$ for $\mathcal{H}^{n-1}$-a.e. $x \in M$, where $\delta$ is constant and $\nu$ is normal to $M$.

Therefore we obtain

$$
\int_{\partial \Omega} \tau(|\operatorname{tr}(u)|) d \mathcal{H}^{n-1}=\int_{M} \tau(|\operatorname{tr}(u)|) d \mathcal{H}^{n-1} \leq \int_{M} \tau\left(c \sum_{i=1}^{n}\left|\operatorname{tr}(u) \cdot \eta_{i}\right|\right) d \mathcal{H}^{n-1},
$$

where $c<+\infty$ depends only on $\left(\eta_{i}\right)_{i=1}^{n}$. The very definition of $\tau$ implies that

$$
\int_{M} \tau\left(c \sum_{i=1}^{n}\left|\operatorname{tr}(u) \cdot \eta_{i}\right|\right) d \mathcal{H}^{n-1} \leq \int_{M} c \sum_{i=1}^{n}\left|\tau\left(\operatorname{tr}(u) \cdot \eta_{i}\right)\right| d \mathcal{H}^{n-1},
$$

where the constant $c<+\infty$ can possibly change from the first to the second term.

Since $\tau\left(\operatorname{tr}(u) \cdot \eta_{i}\right)=\operatorname{tr}\left(\tau\left(u \cdot \eta_{i}\right)\right)$ by the definition of trace in $G S B D(\Omega)$ (see [17, Proof of Theorem $5.2])$, we deduce by (2.13) and (2.14) that

$$
\int_{\partial \Omega} \tau(|\operatorname{tr}(u)|) d \mathcal{H}^{n-1} \leq c \sum_{i=1}^{n} \int_{M}\left|\operatorname{tr}\left(\tau\left(u \cdot \eta_{i}\right)\right)\right| d \mathcal{H}^{n-1} .
$$

We observe now that $\tau\left(u \cdot \eta_{i}\right)$ belongs to $L^{1}(\Omega)$ and its derivative $D_{\eta_{i}} \tau\left(u \cdot \eta_{i}\right)$ belongs to $\mathcal{M}_{b}^{+}(\Omega)$, so that [24, Lemma 1.1] yields

$$
\begin{aligned}
c \sum_{i=1}^{n} \int_{M}\left|\operatorname{tr}\left(\tau\left(u \cdot \eta_{i}\right)\right)\right| d \mathcal{H}^{n-1} & =c \sum_{i=1}^{n} \int_{\partial \Omega}\left|\operatorname{tr}\left(\tau\left(u \cdot \eta_{i}\right)\right)\right| d \mathcal{H}^{n-1} \\
& \leq c \sum_{i=1}^{n} c\left(\Omega, \eta_{i}\right)\left(\left\|\tau\left(u \cdot \eta_{i}\right)\right\|_{L^{1}(\Omega)}+\left|D_{\eta_{i}} \tau\left(u \cdot \eta_{i}\right)\right|(\Omega)\right) \\
& \leq c\left(\|u\|_{L^{1}\left(\Omega, \mathbb{R}^{n}\right)}+\lambda_{u}(\Omega)\right)
\end{aligned}
$$

where $c<+\infty$ depends on $\Omega$ and $\lambda_{u} \in \mathcal{M}_{b}^{+}(\Omega)$ satisfies (2.3). Inequality (2.10) follows from (2.15) and (2.16).

Remark 2.5. Let $u \in G S B D(\Omega) \cap L^{1}\left(\Omega, \mathbb{R}^{n}\right)$ with $\mathcal{H}^{n-1}\left(J_{u}\right)<+\infty$ and let us define

$$
\tilde{\lambda}_{u}(B):=\int_{B}|e(u)| d x+\mathcal{H}^{n-1}\left(J_{u} \cap B\right),
$$

for every $B \subset \Omega$ Borel set. Then (2.6), (2.8), and the coarea formula imply that $\tilde{\lambda}_{u}$ satisfies (2.4).

The following theorem concerns the continuity of the trace operator. For the proof we follow the lines of [23, Section 3.2].

Theorem 2.6 (Continuity of the trace). Assume that $\Omega$ has Lipschitz boundary. Let $u_{k}, u$ belong to $G S B D(\Omega) \cap L^{1}\left(\Omega, \mathbb{R}^{n}\right)$ with $\mathcal{H}^{n-1}\left(J_{u_{k}}\right), \mathcal{H}^{n-1}\left(J_{u}\right)<+\infty$, and let

$$
u_{k} \rightarrow u \quad \text { in } L^{1}\left(\Omega, \mathbb{R}^{n}\right) \quad \text { and } \quad \tilde{\lambda}_{u_{k}} \rightarrow \tilde{\lambda}_{u} \quad \text { weakly* in }\left(C_{b}^{0}\right)^{\prime},
$$


where $\tilde{\lambda}$ has been introduced in (2.17) and the weakly* convergence in $\left(C_{b}^{0}\right)^{\prime}$ means, by definition, that

$$
\int_{\Omega} \varphi d \tilde{\lambda}_{u_{k}} \rightarrow \int_{\Omega} \varphi d \tilde{\lambda}_{u}
$$

for every bounded continuous function $\varphi$ defined on $\Omega$. Then

$$
\int_{\partial \Omega}\left|\operatorname{tr}\left(u_{k}\right)-\operatorname{tr}(u)\right| \wedge 1 d \mathcal{H}^{n-1} \rightarrow 0 .
$$

Proof. Let $\eta>0$ and let $\Omega_{0} \subset \subset \Omega$ be such that

$$
\tilde{\lambda}_{u}\left(\Omega \backslash \bar{\Omega}_{0}\right) \leq \eta \quad \text { and } \quad \tilde{\lambda}_{u}\left(\partial \Omega_{0}\right)=0 .
$$

Let $\varphi_{0} \in C_{c}^{\infty}(\Omega)$ be such that $\varphi_{0}=1$ on $\Omega_{0}$ and $0 \leq \varphi \leq 1$, and let $\psi_{0}:=1-\varphi_{0}$.

By (2.18) and (2.20) we obtain for $k$ large

$$
\begin{gathered}
\int_{\Omega}\left|u_{k}-u\right| d x \leq \frac{\eta}{1+\left\|\psi_{0}\right\|_{L^{\infty}(\Omega)}} \\
\tilde{\lambda}_{u_{k}}\left(\Omega \backslash \bar{\Omega}_{0}\right) \leq \tilde{\lambda}_{u}\left(\Omega \backslash \bar{\Omega}_{0}\right)+\eta \leq 2 \eta .
\end{gathered}
$$

Applying inequality (2.10) to the function $\left(u_{k}-u\right) \psi_{0}$ we find

$$
\begin{aligned}
& \int_{\partial \Omega} \tau\left(\left|\operatorname{tr}\left(u_{k}\right)-\operatorname{tr}(u)\right|\right) d \mathcal{H}^{n-1} \leq \\
\leq \quad & c\left(\left\|\left(u_{k}-u\right) \psi_{0}\right\|_{L^{1}\left(\Omega, \mathbb{R}^{n}\right)}+\int_{\Omega}\left|e\left(\left(u_{k}-u\right) \psi_{0}\right)\right| d x+\mathcal{H}^{n-1}\left(J_{\left(u_{k}-u\right) \psi_{0}}\right)\right) \\
\leq \quad & c\left(\left\|u_{k}-u\right\|_{L^{1}\left(\Omega, \mathbb{R}^{n}\right)}+\int_{\Omega \backslash \bar{\Omega}_{0}}\left|e\left(u_{k}\right)\right| d x+\int_{\Omega \backslash \bar{\Omega}_{0}}|e(u)| d x+\left\|u_{k}-u\right\|_{L^{1}\left(\Omega, \mathbb{R}^{n}\right)}\left\|\psi_{0}\right\|_{L^{\infty}(\Omega)}\right. \\
& \left.+\mathcal{H}^{n-1}\left(J_{u_{k}} \cap\left(\Omega \backslash \bar{\Omega}_{0}\right)\right)+\mathcal{H}^{n-1}\left(J_{u} \cap\left(\Omega \backslash \bar{\Omega}_{0}\right)\right)\right) \\
\leq & c\left(\left\|u_{k}-u\right\|_{L^{1}\left(\Omega, \mathbb{R}^{n}\right)}\left(1+\left\|\psi_{0}\right\|_{L^{\infty}(\Omega)}\right)+\tilde{\lambda}_{u_{k}}\left(\Omega \backslash \bar{\Omega}_{0}\right)+\tilde{\lambda}_{u}\left(\Omega \backslash \bar{\Omega}_{0}\right)\right) \leq 4 c \eta,
\end{aligned}
$$

where in last inequalities we have used (2.20)-(2.22). Since $\eta>0$ is arbitrary we deduce that $\tau\left(\left|\operatorname{tr}\left(u_{k}\right)-\operatorname{tr}(u)\right|\right) \rightarrow 0$ in $L_{\mathcal{H}^{n-1}}^{1}(\partial \Omega)$. Finally using the dominated convergence theorem we obtain (2.19).

\section{The Main Results}

In this section we present the main result of the paper: the approximation theorem for $G S B D$ functions. The application to the Ambrosio-Tortorelli convergence will be given in Section 4 .

Theorem 3.1 (Density). Assume that $\Omega$ has Lipschitz boundary. Let $u \in G S B D^{2}(\Omega) \cap L^{2}\left(\Omega, \mathbb{R}^{n}\right)$. Then there exists a sequence $\left(u_{k}\right) \subset S B V^{2}\left(\Omega, \mathbb{R}^{n}\right) \cap L^{\infty}\left(\Omega, \mathbb{R}^{n}\right)$ such that each $J_{u_{k}}$ is contained in the union $S_{k}$ of a finite number of closed connected pieces of $C^{1}$-hypersurfaces, each $u_{k}$ belongs to $W^{1, \infty}\left(\Omega \backslash S_{k}, \mathbb{R}^{n}\right)$, and the following properties hold:

(1) $\left\|u_{k}-u\right\|_{L^{2}\left(\Omega, \mathbb{R}^{n}\right)} \rightarrow 0$,

(2) $\left\|e\left(u_{k}\right)-e(u)\right\|_{L^{2}\left(\Omega, \mathbb{M}_{s y m}^{n \times n}\right)} \rightarrow 0$,

(3) $\mathcal{H}^{n-1}\left(J_{u_{k}} \triangle J_{u}\right) \rightarrow 0$,

(4) $\int_{J_{u_{k}} \cup J_{u}}\left|u_{k}^{ \pm}-u^{ \pm}\right| \wedge 1 d \mathcal{H}^{n-1} \rightarrow 0$.

We remark that Theorem 3.1 can be combined with the following theorem by Cortesani and Toader [15, Theorem 3.1] (see also [14]) to obtain better approximating functions.

We say that $u \in S B V\left(\Omega, \mathbb{R}^{n}\right)$ is a piecewise smooth $S B V$-function if $u \in W^{m, \infty}\left(\Omega \backslash \overline{J_{u}}, \mathbb{R}^{n}\right)$ for every $m, \mathcal{H}^{n-1}\left(\left(\overline{J_{u}} \cap \Omega\right) \backslash J_{u}\right)=0$, and the set $\overline{J_{u}} \cap \Omega$ is a finite union of closed pairwise disjoint $(n-1)$-simplexes intersected with $\Omega$. 
Theorem 3.2. Assume that $\Omega$ has Lipschitz boundary. Let $u \in S B V^{2}\left(\Omega, \mathbb{R}^{n}\right) \cap L^{\infty}\left(\Omega, \mathbb{R}^{n}\right)$. Then there exists a sequence $\left(u_{k}\right)$ of piecewise smooth $S B V$-functions such that

(1) $\left\|u_{k}-u\right\|_{L^{2}\left(\Omega, \mathbb{R}^{n}\right)} \rightarrow 0$,

(2) $\left\|\nabla u_{k}-\nabla u\right\|_{L^{2}\left(\Omega, \mathbb{M}^{n \times n}\right)} \rightarrow 0$,

(3) $\limsup _{k \rightarrow+\infty} \int_{\bar{A} \cap J_{u_{k}}} \varphi\left(x, u_{k}^{+}, u_{k}^{-}, \nu_{u_{k}}\right) d \mathcal{H}^{n-1} \leq \int_{\bar{A} \cap J_{u}} \varphi\left(x, u^{+}, u^{-}, \nu_{u}\right) d \mathcal{H}^{n-1}$,

for every open set $A \subset \Omega$ and for every upper semicontinuous function $\varphi: \Omega \times \mathbb{R}^{n} \times \mathbb{R}^{n} \times \mathbb{S}^{n-1} \rightarrow[0,+\infty)$ such that

$$
\begin{gathered}
\varphi(x, a, b, \nu)=\varphi(x, b, a,-\nu) \quad \text { for } x \in \Omega, \\
\limsup _{\substack{\left(y, a^{\prime}, b^{\prime}, \mu\right) \rightarrow(x, a, b, \nu) \\
y \in \Omega}} \varphi\left(y, a^{\prime}, b^{\prime}, \mu\right)<+\infty \quad \text { for } x \in \partial \Omega,
\end{gathered}
$$

for every $a, b \in \mathbb{R}^{n}$, and $\nu \in \mathbb{S}^{n-1}$.

Remark 3.3. If $\Omega \subset \mathbb{R}^{n}$ is an open cube, the intersection $\overline{J_{u_{k}}} \cap \Omega$ is a polyhedron, so that the arguments in [15, Remark 3.5] and [14, Corollary 3.11] could be adapted to construct a new approximating sequence $\left(\tilde{u}_{k}\right)$ satisfying all requirements of Theorem 3.2 and for which the set $J_{\tilde{u}_{k}}$ is compactly contained in $\Omega$.

A useful tool for the proof of Theorem 3.1 is the following lemma, which allows us to substitute a $G S B D^{2}$-function with another function of the same type, defined in a larger set, in a way that the norm of the function and of its approximate symmetric gradient, the measure of the jump set, and the trace on $\partial \Omega$ do not increase too much.

Lemma 3.4. Assume that $\Omega$ has Lipschitz boundary. Let $Q: \mathbb{M}_{\text {sym }}^{n \times n} \rightarrow \mathbb{R}$ be a positive definite quadratic form and let $u \in G S B D^{2}(\Omega) \cap L^{2}\left(\Omega, \mathbb{R}^{n}\right)$. Then for every $\varepsilon>0$ we can find a Lipschitz open set $\hat{\Omega}$ with $\Omega \subset \subset \hat{\Omega}$, and a function $\hat{u} \in G S B D^{2}(\hat{\Omega}) \cap L^{2}\left(\hat{\Omega}, \mathbb{R}^{n}\right)$, such that

(1) $\|\hat{u}-u\|_{L^{2}\left(\Omega, \mathbb{R}^{n}\right)}<\varepsilon$,

(2) $\int_{\hat{\Omega}} Q(e(\hat{u})) d x \leq \int_{\Omega} Q(e(u)) d x+\varepsilon$,

(3) $\mathcal{H}^{n-1}\left(J_{\hat{u}}\right) \leq \mathcal{H}^{n-1}\left(J_{u}\right)+\varepsilon$,

(4) $\mathcal{H}^{n-1}\left(J_{\hat{u}} \cap \partial \Omega\right)=0$,

(5) $\int_{\partial \Omega}|\hat{u}-\operatorname{tr}(u)| \wedge 1 d \mathcal{H}^{n-1}<\varepsilon$.

Proof. For the first three properties of the lemma we follow the proof of [12, Lemma 3.2] and we only summarize the essential lines. Property (4) will be an easy consequence of a well-known result in Measure Theory. Eventually, property (5) will be obtained through Theorem 2.6.

Since $\Omega$ has Lipschitz boundary, we can cover $\partial \Omega$ with open sets $\left(A_{i}\right)_{i=1}^{k}$, in a way that each $A_{i} \cap \Omega$ is the subgraph of a Lipschitz function $f_{i}: \Pi^{\xi_{i}} \rightarrow \mathbb{R}$, for a suitable $\xi_{i} \in \mathbb{S}^{n-1}$. Then we consider an open set $A_{0} \subset \subset \Omega$, such that $\bar{\Omega} \subset \bigcup_{i=0}^{k} A_{i}$.

We define

$$
\begin{gathered}
u_{t}^{0}:=u \quad \text { in } A_{0} \\
u_{t}^{i}(x):=u\left(x-t \xi_{i}\right) \quad \text { for } x \in A_{i} \cap\left(\Omega+[0, t) \xi_{i}\right),
\end{gathered}
$$

for $t$ small enough; we extend $u_{t}^{i}$ by 0 in the rest of $A_{i}$.

Clearly we are going to glue the functions $u_{t}^{i}$ together through a partition of unity, but the choice of the partition has to be done properly in view of property (3). 
We choose a partition of unity $\left(\varphi_{i}\right)_{i=0}^{k}$ subordinate to $\left(A_{i}\right)_{i=1}^{k}$ such that $\sum_{i=0}^{k} \varphi_{i}=1$ on $\bar{\Omega}$ and

$$
\mathcal{H}^{n-1}\left(J_{u} \cap \bigcup_{i=0}^{k} \overline{\left\{0<\varphi_{i}<1\right\}}\right) \leq \frac{\varepsilon}{2(k+1)}
$$

this is possible through [12, Lemma 3.3] applied to the positive Borel measure $\mathcal{H}^{n-1}\left\lfloor J_{u}\right.$, which is finite on $\mathbb{R}^{n}$.

We set

$$
u_{\bar{t}}:=\sum_{i=0}^{k} u_{t_{i}}^{i} \varphi_{i} \quad \text { and } \quad \Omega_{\bar{t}}:=A_{0} \cup\left(\bigcup_{i=1}^{k}\left(A_{i} \cap\left(\Omega+\left[0, t_{i}\right) \xi_{i}\right)\right)\right),
$$

where we have set $\bar{t}=\left(t_{1}, \ldots, t_{k}\right)$ and each $t_{i}$ is small. Arguing as in [12, Lemma 3.2] we prove that the pair $\left(u_{\bar{t}}, \Omega_{\bar{t}}\right)$ satisfies properties (1)-(3) for $\bar{t}$ small enough.

Proof of (4). Let us fix $i=1, \ldots, k$, then for every $t \in \mathbb{R}$ we have

$$
\mathcal{H}^{n-1}\left(J_{u_{t}^{i}} \cap \partial \Omega\right)=\mathcal{H}^{n-1}\left(J_{u_{t}^{i}} \cap A_{i} \cap \partial \Omega\right)=\mathcal{H}^{n-1}\left(J_{u} \cap\left(\left(A_{i} \cap \partial \Omega\right)-t \xi_{i}\right)\right) .
$$

Since the measure $\mathcal{H}^{n-1}\left\lfloor J_{u}\right.$ is finite, a classical result of measure theory implies that the pairwise disjoint Borel sets $\left(\left(A_{i} \cap \partial \Omega\right)-t \xi_{i}\right)_{t}$ are $\mathcal{H}^{n-1}\left\lfloor J_{u}\right.$-negligible, except for a countable set of indices $t \in \mathbb{R}$. This proves that $u_{\bar{t}}$ also satisfies property (4) for $\mathcal{L}^{k}$-a.e. $\bar{t} \in \mathbb{R}^{k}$.

Proof of (5). First we note that

$$
\int_{\partial \Omega} \tau\left(\left|\operatorname{tr}\left(u_{\bar{t}}\right)-\operatorname{tr}(u)\right|\right) d \mathcal{H}^{n-1} \leq \sum_{i=1}^{k} \int_{\partial \Omega \cap\left\{\varphi_{i} \neq 0\right\}} \tau\left(\left|\operatorname{tr}\left(u_{t_{i}}^{i}\right)-\operatorname{tr}(u)\right|\right) d \mathcal{H}^{n-1},
$$

where $\tau(s):=\frac{1}{\pi} \operatorname{arctg}(s)$ for $s \in \mathbb{R}$. Let us fix $i=1, \ldots, k$ and let us define $M:=\partial \Omega \cap\left\{\varphi_{i} \neq 0\right\}$. Let $\Omega_{1} \subset \subset A_{i}$ be such that $\partial \Omega_{1}$ is smooth, $M \subset \subset\left(\Omega_{1} \cap \partial \Omega\right)$, and $\mathcal{H}^{n-1}\left(\partial \Omega_{1} \cap J_{u}\right)=0$.

We aim to apply Theorem 2.6 to the functions $u_{t_{i}}^{i}, u$ on the set $\Omega_{1} \cap \Omega$. Clearly we have $u_{t_{i}}^{i} \rightarrow u$ in $L^{1}\left(\Omega_{1} \cap \Omega, \mathbb{R}^{n}\right)$ and $e\left(u_{t_{i}}^{i}\right) \rightarrow e(u)$ in $L^{1}\left(\Omega_{1} \cap \Omega, \mathbb{R}^{n}\right)$ by the $L^{1}$-continuity of the translations. It remains to check that

$$
\int_{J_{u_{i}^{i}} \cap \Omega_{1} \cap \Omega} \psi d \mathcal{H}^{n-1} \rightarrow \int_{J_{u} \cap \Omega_{1} \cap \Omega} \psi d \mathcal{H}^{n-1},
$$

for every $\psi \in C_{b}^{0}\left(\Omega_{1} \cap \Omega\right)$. Fixed $\psi \in C_{b}^{0}\left(\Omega_{1} \cap \Omega\right)$, one easily shows that

$$
\psi\left(x+t_{i} \xi_{i}\right) \chi_{\Omega_{1} \cap \Omega}\left(x+t_{i} \xi_{i}\right) \rightarrow \psi(x) \chi_{\Omega_{1} \cap \Omega}(x)
$$

when $x \in J_{u} \backslash \partial \Omega_{1}$. By our assumptions on $\Omega_{1}$ we find that $\mathcal{H}^{n-1}$-a.e. $x \in J_{u}$ is out of $\partial \Omega_{1}$. By the dominated convergence theorem we eventually obtain (3.3) and finally Theorem 2.6 gives the continuity of the trace. We conclude that there exists $\bar{t}$ small enough such that properties $(1)-(5)$ hold for the pair $\left(u_{\bar{t}}, \Omega_{\bar{t}}\right)$.

The proof of Theorem 3.1 is quite technical, so we break it into three steps. The first step is the following theorem, which will give a rough and unified approximation of the energies.

Theorem 3.5 (A first unified approximation of the energies with bad constants). Assume that $\Omega$ has Lipschitz boundary and let $u \in G S B D^{2}(\Omega) \cap L^{2}\left(\Omega, \mathbb{R}^{n}\right)$. Then there exists a sequence $\left(u_{k}\right) \subset$ $S B V^{2}\left(\Omega, \mathbb{R}^{n}\right) \cap L^{2}\left(\Omega, \mathbb{R}^{n}\right)$ such that $J_{u_{k}}$ is contained in the union $\Sigma_{k}$ of a finite number of $(n-1)$ dimensional closed cubes, $u_{k} \in W^{1, \infty}\left(\Omega \backslash \Sigma_{k}, \mathbb{R}^{n}\right)$, and the following properties hold:

(1) $\left\|u_{k}-u\right\|_{L^{2}\left(\Omega, \mathbb{R}^{n}\right)} \rightarrow 0$,

(2) $\limsup _{k \rightarrow+\infty}\left(\int_{\Omega} Q_{n}\left(e\left(u_{k}\right)\right) d x+\mathcal{H}^{n-1}\left(\Sigma_{k}\right)\right) \leq \int_{\Omega} Q_{n}(e(u)) d x+c_{1} \mathcal{H}^{n-1}\left(J_{u}\right)$. Here $c_{1}$ is a positive constant depending only on the dimension $n$ and $Q_{n}$ is the positive definite quadratic form on $\mathbb{M}_{s y m}^{n \times n}$ defined by

$$
Q_{n}(A):=\frac{3(n-2)}{2} \sum_{i=1}^{n} a_{i, i}^{2}+\operatorname{Tr}\left(A A^{t}\right)+\frac{1}{2}(\operatorname{Tr}(A))^{2}, \quad \text { for } A \in \mathbb{M}_{\text {sym }}^{n \times n},
$$


where $\operatorname{Tr}(A)$ denotes the trace of the matrix $A$;

(3) $\int_{\partial \Omega}\left|\operatorname{tr}\left(u_{k}\right)-\operatorname{tr}(u)\right| \wedge 1 d \mathcal{H}^{n-1} \rightarrow 0$,

(4) if $\left(\Gamma_{i}\right)_{i=1}^{+} \infty$ is a fixed sequence of $C^{1}$-manifolds contained in $\Omega$, then $\left(u_{k}\right)$ can be chosen such that also $\mathcal{H}^{n-1}\left(\Sigma_{k} \cap \Gamma_{i}\right)=0$, for $i=1, \ldots,+\infty$.

Proof. We follow the lines of [12, Proof of Theorem 1]. We first substitute the function $u$ with a similar function $\hat{u}$ defined on a larger set $\hat{\Omega}$. Then we discretize $\hat{u}$ on a suitable lattice and interpolate it with a continuous function. Finally the approximating function will be obtained redefining the interpolating function on some cubes of the lattice which intersect $J_{\hat{u}}$.

Let $u \in G S B D^{2}(\Omega) \cap L^{2}\left(\Omega, \mathbb{R}^{n}\right)$, let $\varepsilon>0$, and let $\hat{u}$ and $\hat{\Omega}$ as in Lemma 3.4. By Lemma 2.2 we can find a basis $e_{1}, \ldots, e_{n}$ of $\mathbb{R}^{n}$ such that, for every vector $e$ in the set

$$
D:=\left\{e_{i}, i=1, \ldots, n, e_{i} \pm e_{j}, 1 \leq i<j \leq n\right\}
$$

one has

$$
\mathcal{H}^{n-1}\left(\left\{x \in J_{\hat{u}}:[\hat{u}](x) \cdot e=0\right\}\right)=0 .
$$

For each small discretization step $h>0$ and for each $y \in[0,1)^{n}$, we define the discretized function of $\hat{u}$

$$
\hat{u}_{h}^{y}(\xi):=\hat{u}(h y+\xi), \quad \text { for } \xi \in h \mathbb{Z}^{n} \cap(\hat{\Omega}-h y) .
$$

We also define the continuous interpolation of $\hat{u}_{h}^{y}$

where

$$
w_{h}^{y}(x):=\sum_{\xi \in h \mathbb{Z}^{n} \cap \hat{\Omega}} \hat{u}_{h}^{y}(\xi) \Delta\left(\frac{x-(\xi+h y)}{h}\right) \quad \text { for } x \in \Omega
$$

$$
\Delta(x):=\prod_{i=1}^{n}\left(1-\left|x_{i}\right|\right)^{+}
$$

We note that $w_{h}^{y} \in W^{1, \infty}\left(\Omega, \mathbb{R}^{n}\right)$. In view of the definition of the discrete energies we introduce

$$
\begin{gathered}
J^{\tau}:=\bigcup_{x \in J_{\hat{u}}}[x, x-\tau] \quad \text { for } \tau \in \mathbb{R}^{n}, \\
l_{e, h}^{y}(\xi):=\chi_{J^{h e}}(h y+\xi) \quad \text { for } \xi \in h \mathbb{Z}^{n} \text { and } e \in D .
\end{gathered}
$$

In what follows $\xi$ is intended to belong to $h \mathbb{Z}^{n}$.

We are now in a position to define the discrete energies

$$
\begin{gathered}
E_{1}^{y, h}(\hat{\Omega}):=h^{n} \sum_{e \in D} \sum_{\substack{\xi \in \hat{\Omega}-h y \\
\xi \in \hat{\Omega}-h y-h e}} \alpha(e) \frac{\left(\left(\hat{u}_{h}^{y}(\xi+h e)-\hat{u}_{h}^{y}(\xi)\right) \cdot e\right)^{2}}{h^{2}}\left(1-l_{e, h}^{y}(\xi)\right), \\
E_{2}^{y, h}(\hat{\Omega}):=\tilde{c}_{1} h^{n} \sum_{e \in D} \sum_{\substack{\xi \in \hat{\Omega}-h y \\
\xi \in \hat{\Omega}-h y-h e}} \frac{l_{e, h}^{y}(\xi)}{|e| h},
\end{gathered}
$$

where $(\alpha(e))_{e \in D}$ are positive parameters, chosen in a way that we shall be able to keep the constant 1 for the bulk term in estimate (2). Precisely, we define $\alpha(e):=n-1$ if $e=e_{i}$, for $i=1, \ldots, n$ and $\alpha(e):=1 / 4$ for $1 \leq i<j \leq n$. Moreover $\tilde{c}_{1}$ is a constant depending only on the dimension $n$ which will be chosen later. We also set $\hat{e}:=e /|e|$.

The first part of the proof is devoted to the choice of a suitable $y \in[0,1)^{n}$, and a suitable subsequence of $h$, not relabelled, such that the following properties hold:

$\left(1^{\prime}\right) \quad\left\|w_{h}^{y}-\hat{u}\right\|_{L^{2}\left(\Omega, \mathbb{R}^{n}\right)} \rightarrow 0$,

$\left(2^{\prime}\right) \quad \lim _{h \rightarrow+\infty}\left[E_{1}^{y, h}(\hat{\Omega})+E_{2}^{y, h}(\hat{\Omega})\right] \leq \int_{\hat{\Omega}} Q_{n}(e(\hat{u})) d x+c_{1} \mathcal{H}^{n-1}\left(J_{\hat{u}}\right)$, where $c_{1}<+\infty$ depends on $\tilde{c}_{1}$, 
$\left(3^{\prime} \mathrm{a}\right) \int_{\partial \Omega}\left|w_{h}^{y}-\hat{u}\right| \wedge 1 d \mathcal{H}^{n-1} \rightarrow 0$

$\left(3^{\prime} \mathrm{b}\right) E_{2}^{y, h}\left((\partial \Omega)_{n h}\right) \rightarrow 0$. Here $(\partial \Omega)_{n h}:=\left\{x \in \mathbb{R}^{n}: \mathrm{d}(x, \partial \Omega)<n h\right\}$ and the expression $E_{2}^{y, h}\left((\partial \Omega)_{n h}\right)$ means that $(\partial \Omega)_{n h}$ replaces $\hat{\Omega}$ in the definition (3.6);

$\left(4^{\prime}\right) \quad$ if $\left(\Gamma_{i}\right)_{i=1}^{+} \infty$ is a fixed sequence of $C^{1}$-manifold contained in $\Omega$, then $y$ and the subsequence of $h$ can be chosen such that also $\mathcal{H}^{n-1}\left(\left(h y+h \mathbb{Z}^{n}+[0, h) e_{j}\right) \cap \Gamma_{i}\right)=0$, for $i=1, \ldots,+\infty$ and $j=1, \ldots, n$.

The first part of the proof (properties $\left(1^{\prime}\right)$ and $\left.\left(2^{\prime}\right)\right)$ is analogous to that in $[12$, Theorem 1$]$. We summarize it for completeness and for future convenience.

Proof of $\left(1^{\prime}\right)$. By the very definition of $w_{h}^{y}$, the Fubini Theorem, and a change of variable we find

$$
\begin{aligned}
\int_{[0,1)^{n}} d y \int_{\Omega}\left|w_{h}^{y}(x)-\hat{u}(x)\right|^{2} d x & \leq \int_{[0,1)^{n}} d y \int_{\Omega} \sum_{\xi \in h \mathbb{Z}^{n} \cap \hat{\Omega}} \Delta\left(\frac{x-(\xi+h y)}{h}\right)|\hat{u}(\xi+h y)-\hat{u}(x)|^{2} d x \\
& \leq \sum_{\xi \in h \mathbb{Z}^{n} \cap \hat{\Omega}} \int_{\Omega} d x \int_{\frac{x-\xi}{h}-[0,1)^{n}} \Delta(z)|\hat{u}(x-h z)-\hat{u}(x)|^{2} d z \\
& \leq \int_{(-1,1)^{n}} \Delta(z) d z \int_{\Omega}|\hat{u}(x-h z)-\hat{u}(x)|^{2} d x
\end{aligned}
$$

where to infer the last inequality we notice that the sets $\frac{x-\xi}{h}-[0,1)^{n}$ are pairwise disjoint as $\xi$ varies in $h \mathbb{Z}^{n} \cap \hat{\Omega}$. The last term in the previous inequality converges to 0 by the dominated convergence theorem. Then property $\left(1^{\prime}\right)$ is satisfied for a subsequence of $h$, not relabelled, and for $y$ varying in a subset of $[0,1)^{n}$ with full measure.

Proof of $\left(2^{\prime}\right)$. Let us estimate

$$
\int_{[0,1)^{n}} E_{j}^{y, h}(\hat{\Omega}) d y
$$

for $j=1,2$. For convenience we introduce $I_{z}^{e}:=\{s \in \mathbb{R}: z+s \hat{e} \in \hat{\Omega}\}$ and $I_{z, h}^{e}:=\{s \in \mathbb{R}: z+s \hat{e} \in$ $\hat{\Omega}, z+(s+h|e|) \hat{e} \in \hat{\Omega}\}$. First a change of variable gives

$$
\begin{aligned}
& \int_{[0,1)^{n}} E_{1}^{y, h}(\hat{\Omega}) d y= \\
= & \sum_{e \in D} \alpha(e) \sum_{\xi \in h \mathbb{Z}^{n}} \int_{\xi+h[0,1)^{n}} \chi_{\hat{\Omega} \cap(\hat{\Omega}-h e)}(x) \frac{|(\hat{u}(x+h e)-\hat{u}(x)) \cdot e|^{2}}{h^{2}}\left(1-\chi_{J^{h e}}(x)\right) d x \\
= & \sum_{e \in D} \alpha(e) \int_{\Pi^{e}} d z \int_{I_{z, h}^{e}} \frac{\left|\hat{u}_{z}^{e}(s+h|e|)-\hat{u}_{z}^{e}(s)\right|^{2}}{h^{2}}\left(1-\chi_{J^{h e}}(z+s \hat{e})\right) d s .
\end{aligned}
$$

As in the $S B D$-case [12], when $\hat{u} \in G S B D^{2}(\hat{\Omega}) \cap L^{2}\left(\hat{\Omega}, \mathbb{R}^{n}\right)$ the slice $\hat{u}_{z}^{e}(s):=\hat{u}(z+s \hat{e}) \cdot \hat{e}$ belongs to $S B V^{2}\left(I_{z}^{e}\right)$, for $e \in D$ and for $\mathcal{H}^{n-1}$-a.e. $z \in \Pi^{e}$. Noticing that $\chi_{J^{h e}}(z+s \hat{e})=0$ is equivalent to $J_{\hat{u}_{z}^{e}} \cap[s, s+h|e|]=0$, we deduce that (3.8) is less than or equal to

$$
\sum_{e \in D} \alpha(e) \int_{\Pi^{e}} d z \int_{I_{z}^{e}}\left|\frac{\partial \hat{u}_{z}^{e}}{\partial s}(t)\right|^{2} d t \leq \int_{\hat{\Omega}} \sum_{e \in D} \alpha(e)|e(\hat{u}) e \cdot e|^{2} d x
$$

where we have used (2.8). Eventually the very definitions of $\alpha(e)$ and $Q_{n}$ give

$$
\sum_{e \in D} \alpha(e)|e(\hat{u}) e \cdot e|^{2}=Q_{n}(e(\hat{u}))
$$

so that

$$
\int_{[0,1)^{n}} E_{1}^{y, h}(\hat{\Omega}) d y \leq \int_{\hat{\Omega}} Q_{n}(e(\hat{u})) d x
$$


The same argument applied to $E_{2}^{y, h}$ gives

$$
\int_{[0,1)^{n}} E_{2}^{y, h}(\hat{\Omega}) d y=\sum_{e \in D} \tilde{c}_{1} \int_{\Pi^{e}} d z \int_{I_{z, h}^{e}} \frac{\chi_{J^{h e}(z+s \hat{e})}}{|e| h} d s \leq \sum_{e \in D} \tilde{c}_{1} \mathcal{H}^{0}\left(J_{\hat{u}_{z}^{e}}\right) \leq c_{1} \mathcal{H}^{n-1}\left(J_{\hat{u}}\right)
$$

where $c_{1}:=\tilde{c}_{1} \max _{|\nu|=1}\left(\sum_{e \in D}|\nu \cdot e| /|e|\right)$ and we have used (2.6).

For technical reasons, which will be clear at the end of the proof, it is convenient to prove properties $\left(3^{\prime} \mathrm{a}\right)-\left(4^{\prime}\right)$ before completing the proof of $\left(2^{\prime}\right)$.

Proof of $\left(3^{\prime}\right.$ a). Using the very definition of $w_{h}^{y}$ and defining $z:=(x-\xi) / h-y$ we obtain

$$
\begin{aligned}
& \int_{[0,1)^{n}} d y \int_{\partial \Omega}\left|w_{h}^{y}(x)-\hat{u}(x)\right| \wedge 1 d \mathcal{H}^{n-1}(x) \leq \\
\leq & \sum_{\xi \in h \mathbb{Z}^{n} \cap \hat{\Omega}} \int_{[0,1)^{n}} d y \int_{\partial \Omega \cap\left(\xi+h y+h(-1,1)^{n}\right)}|\hat{u}(\xi+h y)-\hat{u}(x)| \wedge 1 d \mathcal{H}^{n-1}(x) \\
\leq & \sum_{\xi \in h \mathbb{Z}^{n} \cap \hat{\Omega}} \int_{\partial \Omega \cap\left(\xi+h(-1,2)^{n}\right)} d \mathcal{H}^{n-1}(x) \int_{\frac{x-\xi}{h}-[0,1)^{n}}|\hat{u}(x-h z)-\hat{u}(x)| \wedge 1 d z \\
\leq & \sum_{\xi \in h \mathbb{Z}^{n} \cap \hat{\Omega}} \int_{\partial \Omega \cap\left(\xi+h(-1,2)^{n}\right)} d \mathcal{H}^{n-1}(x) \int_{(-2,2)^{n}}|\hat{u}(x-h z)-\hat{u}(x)| \wedge 1 d z \\
\leq & c \int_{\partial \Omega} d \mathcal{H}^{n-1}(x) f_{B(x, c h)}\left|\hat{u}\left(x^{\prime}\right)-\hat{u}(x)\right| \wedge 1 d x^{\prime},
\end{aligned}
$$

where $c<+\infty$ depends only on the dimension $n$.

Now, for $\mathcal{H}^{n-1}$-a.e. $x \in \partial \Omega$ we obtain

$$
f_{B(x, c h)}\left|\hat{u}\left(x^{\prime}\right)-\hat{u}(x)\right| \wedge 1 d x^{\prime} \rightarrow 0,
$$

by [17, Theorem 5.1] and property (4) of Lemma 3.4 applied to $\hat{u}$. Eventually the dominated convergence theorem implies $\int_{\partial \Omega}\left|w_{h}^{y}(x)-\hat{u}(x)\right| \wedge 1 d \mathcal{H}^{n-1}(x) \rightarrow 0$ in $L^{1}\left([0,1)^{n}\right)$.

Hence property $\left(3^{\prime} \mathrm{a}\right)$ holds for a subsequence of $h$, not relabelled, and $y$ in a subset of $[0,1)^{n}$ with full measure.

Proof of $\left(3^{\prime} b\right)$. This step requires a computation analogous to that in (3.11), which leads to

$$
\int_{[0,1)^{n}} E_{2}^{y, h}\left((\partial \Omega)_{n h}\right) d y \leq c_{1} \mathcal{H}^{n-1}\left(J_{\hat{u}} \cap(\partial \Omega)_{n h}\right) .
$$

Since $\hat{u}$ satisfies property (4) of Lemma 3.4, we find that $E_{2}^{y, h}\left((\partial \Omega)_{n h}\right)$ converges to 0 in $L^{1}\left([0,1)^{n}\right)$ and then a subsequence of $h$ and a set of full measure of $[0,1)^{n}$ satisfy $\left(3^{\prime} \mathrm{b}\right)$.

Proof of $\left(4^{\prime}\right)$. Let us fix $i=1, \ldots,+\infty, j=1, \ldots, n$, and let us consider the set

$$
\Gamma_{i} \cap \bigcup_{\substack{y_{j} \in[0,1) \\ \xi_{j} \in h \mathbb{Z}}}\left\{x \in \mathbb{R}^{n}: x_{j}=h y_{j}+\xi_{j}\right\} .
$$

Since $\bigcup_{\xi_{j} \in h \mathbb{Z}}\left\{x \in \mathbb{R}^{n}: x_{j}=h y_{j}+\xi_{j}\right\}$ are disjoint sets as $y_{j}$ varies in $[0,1)$ and since the measure $\mathcal{H}^{n-1}\left\lfloor\Gamma_{i}\right.$ is finite, we infer for $\mathcal{H}^{n-1}$-a.e. $y_{j} \in[0,1)$ the following holds

$$
\mathcal{H}^{n-1}\left(\bigcup_{\xi_{j} \in h \mathbb{Z}}\left(\Gamma_{i} \cap\left\{x \in \mathbb{R}^{n}: x_{j}=h y_{j}+\xi_{j}\right\}\right)\right)=0 .
$$

Taking the union as $i=1, \ldots,+\infty$ and $j=1, \ldots, n$ we obtain $\left(4^{\prime}\right)$.

Continuation of the proof of $\left(2^{\prime}\right)$. Let us consider the subsequence of $h$ given by the proofs of $\left(1^{\prime}\right),\left(3^{\prime} \mathrm{a}\right),\left(3^{\prime} \mathrm{b}\right)$, and $\left(4^{\prime}\right)$ and write inequalities (3.10) and (3.11) for this subsequence. Now we are 
in the position to apply the Fatou Lemma, so that

$$
\int_{[0,1)^{n}} \liminf _{h \rightarrow 0}\left[E_{1}^{y, h}(\hat{\Omega})+E_{2}^{y, h}(\hat{\Omega})\right] d y \leq \int_{\hat{\Omega}} Q_{n}(e(\hat{u})) d x+c_{1} \mathcal{H}^{n-1}\left(J_{\hat{u}}\right) .
$$

Eventually we can find $y \in[0,1)^{n}$ and a further subsequence of $h$, not relabelled, such that properties $\left(1^{\prime}\right)-\left(4^{\prime}\right)$ hold. In what follows we shall omit $y$, writing, e.g., $w_{h}$ in place of $w_{h}^{y}$.

In this second part of the proof we redefine the function $w_{h}$ within some cubes. Precisely, we say that a hypercube

$$
C=\xi+h y+[0, h)^{n}
$$

is "bad" if either $J_{\hat{u}}$ crosses an edge of $C$

$$
\xi+h y+h \eta+\left[0, h e_{i}\right], \text { where } i=1, \ldots, n \text { and } \eta \in\{0,1\}^{n} \text { with } \eta_{i}=0
$$

(namely if $l_{e_{i}, h}(\xi+h \eta)=\chi_{J^{h e_{i}}}(\xi+h y+h \eta)=1$ ), or $J_{\hat{u}}$ crosses a diagonal of a 2-dimensional face

$$
\xi+h y+h \eta+\left[0, h\left(e_{i}+e_{j}\right)\right], \text { where } i<j \text { and } \eta \in\{0,1\}^{n} \text { with } \eta_{i}=\eta_{j}=0
$$

(namely if $\left.l_{e_{i}+e_{j}, h}(\xi+h \eta)=\chi_{J^{h\left(e_{i}+e_{j}\right)}}(\xi+h y+h \eta)=1\right)$, or

$$
\xi+h y+h \eta+\left[h e_{j}, h e_{j}+h\left(e_{i}-e_{j}\right)\right] \text {, where } i<j \text { and } \eta \in\{0,1\}^{n} \text { with } \eta_{i}=\eta_{j}=0
$$

(namely if $l_{e_{i}-e_{j}, h}\left(\xi+h \eta+h e_{j}\right)=\chi_{J^{h\left(e_{i}-e_{j}\right)}}\left(\xi+h y+h \eta+h e_{j}\right)=1$ ). We define $v_{h}:=0$ in every bad hypercube and $v_{h}:=w_{h}$ otherwise.

Thanks to the previous definition the following properties hold:

$\left(1^{\prime \prime}\right) \quad\left\|w_{h}-v_{h}\right\|_{L^{2}\left(\Omega, \mathbb{R}^{n}\right)} \rightarrow 0$,

$\left(2^{\prime \prime}\right)$ the constant $\tilde{c}_{1}(n)$ in $(3.6)$ can be chosen in a way that

$$
\int_{\Omega} Q_{n}\left(e\left(v_{h}\right)\right) d x+\mathcal{H}^{n-1}\left(\overline{J_{v_{h}}}\right) \leq E_{1}^{y, h}(\hat{\Omega})+E_{2}^{y, h}(\hat{\Omega}),
$$

$\left(3^{\prime \prime}\right) \quad \int_{\partial \Omega}\left|w_{h}-\operatorname{tr}\left(v_{h}\right)\right| \wedge 1 d \mathcal{H}^{n-1} \rightarrow 0$, where $\operatorname{tr}\left(v_{h}\right)$ is the trace from the interior of $\Omega$.

The proof of $\left(1^{\prime \prime}\right)$ and of $\left(2^{\prime \prime}\right)$ work as in $[12,13]$ since the definition of $v_{h}$ and of the discrete energies are the same. Let us prove now $\left(3^{\prime \prime}\right)$.

Proof of $\left(3^{\prime \prime}\right)$. First we note that

$$
\int_{\partial \Omega}\left|w_{h}-\operatorname{tr}\left(v_{h}\right)\right| \wedge 1 d \mathcal{H}^{n-1} \leq \mathcal{H}^{n-1}\left(\left\{\partial \Omega \cap \bigcup_{C \text { bad cube }} C\right\}\right)
$$

and that for each cube we have

$$
\mathcal{H}^{n-1}(\{\partial \Omega \cap C\}) \leq \operatorname{ch}^{n-1},
$$

where $c$ depends on $\Omega$. Now the contribution of a bad cube $C$ to $E_{2}^{h}\left((\partial \Omega)_{n h}\right)$ is given by

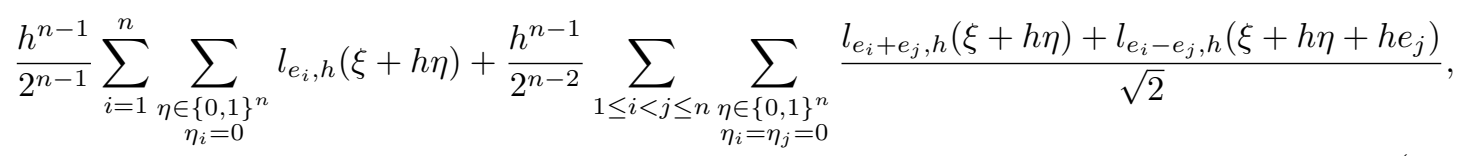

where the coefficients take into account the fact that each edge is common to $2^{n-1}$ hypercubes and a diagonal of a 2 -face is common to $2^{n-2}$ hypercubes. Since at least one of the $l_{e, h}$ in the sum is equal to 1 , we find the term in (3.17) is greater than or equal to $\frac{h^{n-1}}{2^{n-1}}$. Hence by this and (3.16) we find

$$
\sum_{C \text { bad cube }} \mathcal{H}^{n-1}(\{\partial \Omega \cap C\}) \leq c E_{2}^{h}\left((\partial \Omega)_{n h}\right),
$$

for a suitable constant $c<+\infty$ depending on $\Omega$. Thanks to property $\left(3^{\prime} \mathrm{b}\right)$ we eventually obtain $\left(3^{\prime \prime}\right)$.

Finally properties $\left(1^{\prime}\right)-\left(4^{\prime}\right),\left(1^{\prime \prime}\right)-\left(3^{\prime \prime}\right)$, and $(1)-(5)$ of Lemma 3.4 yield (1)-(4). 
With the next theorem we provide a further approximation of the given function in a way that the unified estimate for the bulk and the surface energies has now the right coefficients. The proof follows the line of [12, Theorem 2].

Theorem 3.6 (A unified approximation of the energies with the right constants). Assume that $\Omega$ has Lipschitz boundary. Let $u \in G S B D^{2}(\Omega) \cap L^{2}\left(\Omega, \mathbb{R}^{n}\right)$. Then there exists a sequence $\left(u_{k}\right) \subset$ $S B V^{2}\left(\Omega, \mathbb{R}^{n}\right) \cap L^{2}\left(\Omega, \mathbb{R}^{n}\right)$ such that $J_{u_{k}}$ is contained in the union $S_{k}$ of a finite number of closed connected pieces of $C^{1}$-hypersurfaces, $u_{k} \in W^{1, \infty}\left(\Omega \backslash S_{k}, \mathbb{R}^{n}\right)$, and the following properties hold:

(1) $\left\|u_{k}-u\right\|_{L^{2}\left(\Omega, \mathbb{R}^{n}\right)} \rightarrow 0$,

(2) $\limsup _{k \rightarrow+\infty}\left(\int_{\Omega} Q_{n}\left(e\left(u_{k}\right)\right) d x+\mathcal{H}^{n-1}\left(S_{k}\right)\right) \leq \int_{\Omega} Q_{n}(e(u)) d x+\mathcal{H}^{n-1}\left(J_{u}\right)$,

(3) $\int_{J_{u}}\left|u_{k}^{ \pm}-u^{ \pm}\right| \wedge 1 d \mathcal{H}^{n-1} \rightarrow 0$,

(4) $\mathcal{H}^{n-1}\left(J_{u} \backslash J_{u_{k}}\right) \rightarrow 0$, where $Q_{n}$ is defined in (3.4).

Proof. Since $J_{u}$ is $\left(\mathcal{H}^{n-1}, n-1\right)$-rectifiable, we can find a sequence $\left(\Gamma_{i}\right)$ of $C^{1}$-hypersurfaces such that $\mathcal{H}^{n-1}\left(J_{u} \backslash \bigcup_{i=1}^{+} \infty \Gamma_{i}\right)=0$. We fix now $\varepsilon>0$ and use a Besicovitch recovering argument, as in [12, Theorem 2], to find a sequence of pairwise disjoint closed balls $\overline{B_{j}} \subset \Omega$ and an index $j_{0}$ such that

(a) for every $j$ there exists $i_{j}$ for which $\Gamma_{i_{j}}$ divides $B_{j}$ into two connected components,

(b) $\mathcal{H}^{n-1}\left(J_{u} \cap \partial B_{j}\right)=0$,

(c) $\mathcal{H}^{n-1}\left(J_{u} \backslash \bigcup_{j \geq 1} \overline{B_{j}}\right)=0$

(d) $\sum_{j>j_{0}} \mathcal{H}^{n-1}\left(J_{u} \cap B_{j}\right)<\varepsilon$,

(e) $\mathcal{H}^{n-1}\left(\left(J_{u} \triangle \Gamma_{i_{j}}\right) \cap \overline{B_{j}}\right) \leq \frac{\varepsilon}{1-\varepsilon} \mathcal{H}^{n-1}\left(J_{u} \cap \overline{B_{j}}\right)$, for $j=1, \ldots, j_{0}$.

Applying Theorem 3.5 in both of connected components of $B_{j} \backslash \Gamma_{i_{j}}$, we find a sequence of functions $u_{k}^{j}$ defined $\mathcal{L}^{n}$-a.e. on $B_{j}$ for which property (1) of Theorem 3.5 holds in $B_{j}$, property (3) holds in $\partial B_{j}$ and in $\Gamma_{i_{j}}$, property (4) holds for the sequence $\left(\Gamma_{i}\right)$ introduced above, and

$$
\begin{aligned}
\limsup _{k \rightarrow+\infty} \int_{B_{j}} Q_{n}\left(e\left(u_{k}^{j}\right)\right) d x+\mathcal{H}^{n-1}\left(\overline{J_{u_{k}^{j}} \cap B_{j}}\right) \leq & \int_{B_{j}} Q_{n}(e(u)) d x+\mathcal{H}^{n-1}\left(J_{u} \cap B_{j}\right) \\
& +c \frac{\varepsilon}{1-\varepsilon} \mathcal{H}^{n-1}\left(J_{u} \cap B_{j}\right),
\end{aligned}
$$

for a suitable universal constant $c<+\infty$.

Defined

we observe that

$$
A_{t}:=\left\{x \in \mathbb{R}^{n}: \operatorname{dist}\left(x, \Omega \backslash \bigcup_{j=1}^{j_{0}} \overline{B_{j}}\right)<t\right\}
$$

$$
\mathcal{H}^{n-1}\left(J_{u} \cap \bigcap_{t>0} A_{t}\right)=\mathcal{H}^{n-1}\left(J_{u} \backslash \bigcup_{j=1}^{j_{0}} \overline{B_{j}}\right)<\varepsilon \quad \text { and } \lim _{t \rightarrow 0} \int_{A_{t} \cap \bigcup_{j=1}^{j_{0}} B_{j}} Q_{n}(e(u)) d x=0,
$$

therefore we can choose $t>0$ such that

$$
\int_{A_{t} \cap \bigcup_{j=1}^{j_{0}} B_{j}} Q_{n}(e(u)) d x<\varepsilon \text { and } \mathcal{H}^{n-1}\left(J_{u} \cap A_{t}\right)<\varepsilon .
$$

Let $\left(u_{k}^{0}\right)$ be the sequence obtained applying Theorem 3.5 in $A_{t} \cap \Omega$. Then using (3.19) we find

$$
\limsup _{k \rightarrow+\infty} \int_{A_{t} \cap \Omega} Q_{n}\left(e\left(u_{k}^{0}\right)\right) d x+\mathcal{H}^{n-1}\left(\overline{J_{u_{k}^{0}}}\right) \leq \int_{A_{t} \cap \Omega} Q_{n}(e(u)) d x+c \varepsilon .
$$


Now we construct a suitable partition of unity to glue together the functions $u_{k}^{j}$. For $j=0, \ldots, j_{0}$ we find a compact set $K_{j}$, with ${\overline{A_{t}}}^{c} \cap B_{j} \subset \subset K_{j} \subset \subset B_{j}$, such that

$$
\mathcal{H}^{n-1}\left(\left(B_{j} \backslash K_{j}\right) \cap \Gamma_{i_{j}}\right)<\frac{\varepsilon}{j_{0}} .
$$

Let $\varphi_{j} \in C_{c}^{\infty}\left(B_{j}\right)$ for $j=1, \ldots, j_{0}$ such that $\varphi_{j}=1$ in $K_{j}$ and $0 \leq \varphi \leq 1$. Let also $\varphi_{0} \in C_{c}^{\infty}\left(A_{t}\right)$ be such that $\varphi_{0}:=1-\varphi_{j}$ in $B_{j}$ and $\varphi_{0}:=1$ in $\Omega \backslash \bigcup_{j=1}^{j_{0}} B_{j}$.

We finally define

$$
u_{k}:=\sum_{j=0}^{j_{0}} \varphi_{j} u_{k}^{j} .
$$

Then property (1) is satisfied by construction. As for property (2), inequalities (3.18), (3.19), and (3.20) yield

$$
\limsup _{k \rightarrow+\infty} \int_{\Omega} Q_{n}\left(e\left(u_{k}\right)\right) d x+\mathcal{H}^{n-1}\left(\overline{J_{u_{k}}}\right) \leq \int_{\Omega} Q_{n}(e(u)) d x+\mathcal{H}^{n-1}\left(\overline{J_{u}}\right)+c \varepsilon,
$$

where $c<+\infty$ is a universal constant.

Let us prove property (3). Using (c), (d), and (e) we find

$$
\begin{aligned}
\int_{J_{u}}\left|u_{k}^{ \pm}-u^{ \pm}\right| \wedge 1 d \mathcal{H}^{n-1} & \leq \int_{J_{u} \cap \bigcup_{j=1}^{j_{0}}\left(B_{j} \cap \Gamma_{i_{j}}\right)}\left|u_{k}^{ \pm}-u^{ \pm}\right| \wedge 1 d \mathcal{H}^{n-1}+c \varepsilon \\
& \leq \sum_{j=1}^{j_{0}} \int_{B_{j} \cap \Gamma_{i_{j}}}\left|u_{k}^{ \pm}-u^{ \pm}\right| \wedge 1 d \mathcal{H}^{n-1}+c \varepsilon .
\end{aligned}
$$

The very definition of $u_{k}$ implies now that (3.22) is less than or equal to

$$
\begin{aligned}
& \sum_{j=1}^{j_{0}} \sum_{l=0}^{j_{0}} \int_{B_{j} \cap \Gamma_{i_{j}}} \varphi_{l}\left|u_{k}^{l \pm}-u^{ \pm}\right| \wedge 1 d \mathcal{H}^{n-1}+c \varepsilon \\
= & \sum_{j=1}^{j_{0}}\left(\int_{B_{j} \cap \Gamma_{i_{j}}} \varphi_{0}\left|u_{k}^{0^{ \pm}}-u^{ \pm}\right| \wedge 1 d \mathcal{H}^{n-1}\right. \\
& \left.+\int_{B_{j} \cap \Gamma_{i_{j}}} \varphi_{j}\left|u_{k}^{j \pm}-u^{ \pm}\right| \wedge 1 d \mathcal{H}^{n-1}\right)+c \varepsilon \\
\leq & \sum_{j=1}^{j_{0}} \int_{B_{j} \cap \Gamma_{i_{j}}}\left|u_{k}^{j \pm}-u^{ \pm}\right| \wedge 1 d \mathcal{H}^{n-1}+c \varepsilon,
\end{aligned}
$$

where $c<+\infty$ and the last two inequalities follow from the assumptions on $\varphi_{j}$ and from (3.21). By the definition of $u_{k}^{j}$, passing to the limit as $k \rightarrow+\infty$ we find

$$
\limsup _{k \rightarrow+\infty} \int_{J_{u}}\left|u_{k}^{ \pm}-u^{ \pm}\right| \wedge 1 d \mathcal{H}^{n-1} \leq c \varepsilon .
$$

Eventually a diagonalization argument conclude the proof of properties (2) and (3).

Now property (4) easily follows from property (3). Indeed, the measure $\mathcal{H}^{n-1}\left\lfloor J_{u}\right.$ is absolutely continuous with respect to the measure defined by

$$
\nu(B):=\int_{B \cap J_{u}}|[u]| \wedge 1 d \mathcal{H}^{n-1},
$$

for every Borel set $B \subset \Omega$. Moreover

$$
\int_{J_{u} \backslash J_{u_{k}}}|[u]| \wedge 1 d \mathcal{H}^{n-1} \rightarrow 0
$$

holds true by property (3); this yields property (4) and concludes the proof. 
We are now in a position to prove the Density Theorem 3.1. The proof follows the lines of $[12$, Theorem 3].

Proof of the Density Theorem 3.1. Let us consider the sequence $\left(u_{k}\right)$ given by Theorem 3.6. Using the compactness result for $G S B D$ [17, Theorem 11.3] we infer that a subsequence of $\left(u_{k}\right)$, not relabelled, satisfies

$$
\begin{gathered}
e\left(u_{k}\right) \rightarrow e(u) \quad \text { weakly in } L^{2}\left(\Omega, \mathbb{M}_{s y m}^{n \times n}\right), \\
\int_{\Omega} Q_{n}(e(u)) d x \leq \liminf _{k \rightarrow+\infty} \int_{\Omega} Q_{n}\left(e\left(u_{k}\right)\right) d x, \\
\mathcal{H}^{n-1}\left(J_{u}\right) \leq \liminf _{k \rightarrow+\infty} \mathcal{H}^{n-1}\left(J_{u_{k}}\right) .
\end{gathered}
$$

From property (2) of Theorem 3.6 and from (3.25) and (3.26) we deduce

$$
\begin{gathered}
\int_{\Omega} Q_{n}(e(u)) d x=\lim _{k \rightarrow+\infty} \int_{\Omega} Q_{n}\left(e\left(u_{k}\right)\right) d x, \\
\mathcal{H}^{n-1}\left(J_{u}\right)=\lim _{k \rightarrow+\infty} \mathcal{H}^{n-1}\left(J_{u_{k}}\right) .
\end{gathered}
$$

Now (3.24) and (3.27) yield property (2) of the thesis. Property (3) follows from property (4) of Theorem 3.6 and from (3.28). To obtain property (4) it is sufficient to use property (3) of Theorem 3.6 and the already proved property $(3)$ of the thesis.

\section{An Application: Approximation of Brittle Fracture Energies}

In this section we compute the $\Gamma$-limit in $L^{1}\left(\Omega, \mathbb{R}^{n}\right) \times L^{1}(\Omega)$ of the sequence of functionals

$$
G_{k}(u, v):= \begin{cases}\int_{\Omega}\left(Q(v, e(u))+\frac{\psi(v)}{\varepsilon_{k}}+a \varepsilon_{k}^{p-1}|\nabla v|^{p}+|u-g|^{2}\right) d x & \text { if }(u, v) \in H^{1}\left(\Omega, \mathbb{R}^{n}\right) \times V_{\eta_{k}}, \\ +\infty & \text { otherwise }\end{cases}
$$

where

(a) $\Omega \subset \mathbb{R}^{n}$ is a bounded open set and $\varepsilon_{k}>0, \eta_{k} \geq 0$ are infinitesimal sequences with $\eta_{k} / \varepsilon_{k} \rightarrow 0$,

(b) $Q: \mathbb{R} \times \mathbb{M}_{\text {sym }}^{n \times n} \rightarrow \mathbb{R}$ is lower semicontinuous,

(c) for every $s \in \mathbb{R}$, the function $Q(s, \cdot)$ is a positive definite quadratic form on $\mathbb{M}_{s y m}^{n \times n}$,

(d) there exist two constants $0<c_{1}, c_{2}<+\infty$, such that $c_{1} s|A|^{2} \leq Q(s, A) \leq c_{2} s|A|^{2}$, for every $s \in \mathbb{R}$ and $A \in \mathbb{M}_{\text {sym }}^{n \times n}$,

(e) $\psi \in C([0,1])$ is strictly decreasing with $\psi(1)=0$ and $g \in L^{2}\left(\Omega, \mathbb{R}^{n}\right)$,

(f) $a, p \in \mathbb{R}$ with $a>0$ and $p>1$

(g) $V_{\eta_{k}}:=\left\{v \in W^{1, p}(\Omega): \eta_{k} \leq v \leq 1 \mathcal{L}^{n}\right.$-a.e. in $\left.\Omega\right\}$.

We also define the functional $\Psi: L^{1}\left(\Omega, \mathbb{R}^{n}\right) \rightarrow[0,+\infty]$ by

$$
\Psi(u):= \begin{cases}\int_{\Omega} Q(e(u)) d x+\alpha \mathcal{H}^{n-1}\left(J_{u}\right)+\int_{\Omega}|u-g|^{2} d x & \text { if } u \in G S B D^{2}(\Omega) \cap L^{2}\left(\Omega, \mathbb{R}^{n}\right), \\ +\infty & \text { otherwise, }\end{cases}
$$

where $Q(e(u)):=Q(1, e(u))$ and

$$
\alpha:=2 q^{\frac{1}{q}}(a p)^{\frac{1}{p}} \int_{0}^{1} \psi^{\frac{1}{q}} d s, \quad \frac{1}{p}+\frac{1}{q}=1 .
$$

Then the following result holds.

Theorem 4.1. Assume (a)-(g) and assume that $\Omega$ has Lipschitz boundary. Then the $\Gamma$-limit of $\left(G_{k}\right)$ in $L^{1}\left(\Omega, \mathbb{R}^{n}\right) \times L^{1}(\Omega)$ is given by

$$
G(u, v):= \begin{cases}\Psi(u) & \text { if } v=1 \mathcal{L}^{n} \text {-a.e. in } \Omega \\ +\infty & \text { otherwise. }\end{cases}
$$

The previous theorem, together with a compactness result for the functionals $G_{k}$ (Proposition $4.5)$, will give in turn the convergence of minima and minimizers in the space $L^{2}\left(\Omega, \mathbb{R}^{n}\right) \times L^{1}(\Omega)$. 
Corollary 4.2. Assume (a)-(g) and assume that $\Omega$ has Lipschitz boundary. For every $k$, let $\left(u_{k}, v_{k}\right)$ be a minimizer of the problem

$$
\min _{(u, v) \in H^{1}\left(\Omega, \mathbb{R}^{n}\right) \times V_{\eta_{k}}} \int_{\Omega}\left(Q(v, e(u))+\frac{\psi(v)}{\varepsilon_{k}}+a \varepsilon_{k}^{p-1}|\nabla v|^{p}+|u-g|^{2}\right) d x .
$$

Then $v_{k} \rightarrow 1$ in $L^{1}(\Omega)$ and a subsequence of $\left(u_{k}\right)$ converges in $L^{2}\left(\Omega, \mathbb{R}^{n}\right)$ to a minimizer $u$ of the following problem

$$
\min _{u \in G S B D(\Omega)}\left(\int_{\Omega} Q(e(u)) d x+\alpha \mathcal{H}^{n-1}\left(J_{u}\right)+\int_{\Omega}|u-g|^{2} d x\right) .
$$

Moreover the minimum values in (4.2) tend to the minimum value in (4.3).

As usual, we shall prove Theorem 4.1 giving a lower estimate for the $\Gamma$-lower limit of $G_{k}$ and an upper estimate for the $\Gamma$-upper limit of $G_{k}$. To simplify the notation we introduce the functionals $F_{k}: L^{1}\left(\Omega, \mathbb{R}^{n}\right) \times L^{1}(\Omega) \rightarrow[0,+\infty]$ and $\Phi: L^{1}\left(\Omega, \mathbb{R}^{n}\right) \rightarrow[0,+\infty]$ defined by

$$
\begin{aligned}
F_{k}(u, v):= \begin{cases}\int_{\Omega}\left(Q(v, e(u))+\frac{\psi(v)}{\varepsilon_{k}}+a \varepsilon_{k}^{p-1}|\nabla v|^{p}\right) d x & \text { if }(u, v) \in H^{1}\left(\Omega, \mathbb{R}^{n}\right) \times V_{\eta_{k}}, \\
+\infty & \text { otherwise, }\end{cases} \\
\Phi(u):= \begin{cases}\int_{\Omega} Q(e(u)) d x+\alpha \mathcal{H}^{n-1}\left(J_{u}\right) & \text { if } u \in G S B D^{2}(\Omega) \cap L^{1}\left(\Omega, \mathbb{R}^{n}\right), \\
+\infty & \text { otherwise. }\end{cases}
\end{aligned}
$$

For technical reasons which will be clear in the last part of the proof, we first study the $\Gamma$-lower limit of $F_{k}$ in the space $L^{1}\left(\Omega, \mathbb{R}^{n}\right) \times L^{1}(\Omega)$ (Theorem 4.3) and the $\Gamma$-upper limit of (the restriction of) $F_{k}$ in the space $L^{2}\left(\Omega, \mathbb{R}^{n}\right) \times L^{1}(\Omega)$ (Theorem 4.4).

Theorem 4.3. Assume $(a)-(g)$. Let $(u, v) \in L^{1}\left(\Omega, \mathbb{R}^{n}\right) \times L^{1}(\Omega)$ and let $\left(u_{k}, v_{k}\right)$ be a sequence such that

$$
\begin{gathered}
\left(u_{k}, v_{k}\right) \rightarrow(u, v) \text { in } L^{1}\left(\Omega, \mathbb{R}^{n}\right) \times L^{1}(\Omega), \\
\left(F_{k}\left(u_{k}, v_{k}\right)\right) \text { is bounded. }
\end{gathered}
$$

Then $u \in G S B D^{2}(\Omega) \cap L^{1}\left(\Omega, \mathbb{R}^{n}\right), v=1 \mathcal{L}^{n}$-a.e. in $\Omega$, and

$$
\begin{gathered}
\int_{\Omega} Q(e(u)) d x \leq \liminf _{k \rightarrow+\infty} \int_{\Omega} Q\left(v_{k}, e\left(u_{k}\right)\right) d x \\
\alpha \mathcal{H}^{n-1}\left(J_{u}\right) \leq \liminf _{k \rightarrow+\infty} \int_{\Omega}\left(\frac{\psi\left(v_{k}\right)}{\varepsilon_{k}}+a \varepsilon_{k}^{p-1}\left|\nabla v_{k}\right|^{p}\right) d x .
\end{gathered}
$$

Proof. The convergence $v_{k} \rightarrow 1$ in $L^{1}(\Omega)$ is an immediate consequence of (4.4) and (4.5). In the first part of the proof we argue by slicing following the lines of [18, Proposition 1].

Proof of (4.6). We fix $\xi \in \mathbb{R}^{n}, \xi \neq 0$. We are going to prove that $u \in G S B D(\Omega)$ and that satisfies

$$
\int_{\Omega}(e(u) \xi \cdot \xi)^{2} d x \leq \liminf _{k \rightarrow+\infty} \int_{\Omega} v_{k}\left(e\left(u_{k}\right) \xi \cdot \xi\right)^{2} d x
$$

To this aim we first extract a subsequence $\left(u_{r}, v_{r}\right)$ of $\left(u_{k}, v_{k}\right)$ such that

$$
\left(\left(u_{r}\right)_{y}^{\xi},\left(v_{r}\right)_{y}^{\xi}\right) \rightarrow\left(u_{y}^{\xi}, 1\right) \text { in } L^{1}\left(\Omega_{y}^{\xi}\right) \times L^{1}\left(\Omega_{y}^{\xi}\right) \text { for } \mathcal{H}^{n-1} \text {-a.e. } y \in \Omega^{\xi}
$$

and

$$
\lim _{r \rightarrow+\infty} \int_{\Omega} v_{r}\left(e\left(u_{r}\right) \xi \cdot \xi\right)^{2} d x=\liminf _{k \rightarrow+\infty} \int_{\Omega} v_{k}\left(e\left(u_{k}\right) \xi \cdot \xi\right)^{2} d x .
$$

Fixed $0<\kappa<1$, the Fubini Theorem, [2, Structure Theorem 4.5], and (4.5) imply

$$
\begin{gathered}
\int_{\Omega^{\xi}}\left[\int_{\Omega_{y}^{\xi}}\left(\left(v_{r}\right)_{y}^{\xi}\left|\nabla\left(\left(u_{r}\right){ }_{y}^{\xi}\right)\right|^{2}+\kappa\left(\frac{\psi\left(v_{r}\right)_{y}^{\xi}}{\varepsilon_{r}}+a \varepsilon_{r}^{p-1}\left|\nabla\left(v_{r}\right)_{y}^{\xi}\right|^{p}\right)\right) d t\right] d \mathcal{H}^{n-1}(y) \leq \\
\leq \int_{\Omega}\left(v_{r}\left(e\left(u_{r}\right) \xi \cdot \xi\right)^{2}+\kappa\left(\frac{\psi\left(v_{r}\right)}{\varepsilon_{r}}+a \varepsilon_{r}^{p-1}\left|\nabla\left(v_{r}\right)\right|^{p}\right)\right) d x \leq c,
\end{gathered}
$$


where $c<+\infty$ is constant. Using the previous inequality and the Fatou Lemma, for $\mathcal{H}^{n-1}$-a.e. $y \in \Omega^{\xi}$ we can find a subsequence $\left(u_{m}, v_{m}\right)$ of $\left(u_{r}, v_{r}\right)$ such that

$$
\begin{aligned}
& \lim _{m \rightarrow+\infty} \int_{\Omega_{y}^{\xi}}\left(\left(v_{m}\right)_{y}^{\xi}\left|\nabla\left(\left(u_{m}\right){ }_{y}^{\xi}\right)\right|^{2}+\kappa\left(\frac{\psi\left(v_{m}\right)_{y}^{\xi}}{\varepsilon_{m}}+a \varepsilon_{m}^{p-1}\left|\nabla\left(v_{m}\right)_{y}^{\xi}\right|^{p}\right)\right) d t= \\
= & \liminf _{r \rightarrow+\infty} \int_{\Omega_{y}^{\xi}}\left(\left(v_{r}\right)_{y}^{\xi}\left|\nabla\left(\left(u_{r}\right)_{y}^{\xi}\right)\right|^{2}+\kappa\left(\frac{\psi\left(v_{r}\right)_{y}^{\xi}}{\varepsilon_{r}}+a \varepsilon_{r}^{p-1}\left|\nabla\left(v_{r}\right)_{y}^{\xi}\right|^{p}\right)\right) d t<+\infty .
\end{aligned}
$$

Since (4.9) and (4.12) hold, we can apply the scalar result [22, Proposition 3.4] to $\left(\left(u_{m}\right)_{y}^{\xi},\left(v_{m}\right)_{y}^{\xi}\right)$, so that $u_{y}^{\xi} \in S B V^{2}\left(\Omega_{y}^{\xi}\right)$ and

$$
\begin{gathered}
\int_{\Omega_{y}^{\xi}}\left|\nabla\left(u_{y}^{\xi}\right)\right|^{2} d t \leq \liminf _{m \rightarrow+\infty} \int_{\Omega_{y}^{\xi}}\left(v_{m}\right)_{y}^{\xi}\left|\nabla\left(\left(u_{m}\right)_{y}^{\xi}\right)\right|^{2} d t \\
\alpha \mathcal{H}^{n-1}\left(J_{u_{y}^{\xi}}\right) \leq \liminf _{m \rightarrow+\infty} \int_{\Omega_{y}^{\xi}}\left(\frac{\psi\left(v_{m}\right)_{y}^{\xi}}{\varepsilon_{m}}+a \varepsilon_{m}^{p-1}\left|\nabla\left(\left(v_{m}\right)_{y}^{\xi}\right)\right|^{p}\right) d t .
\end{gathered}
$$

To check that $u \in G S B D(\Omega)$, we observe the following inequalities hold

$$
\begin{aligned}
& \int_{\Omega^{\xi}}\left(\left|D\left(u_{y}^{\xi}\right)\right|\left(\Omega_{y}^{\xi} \backslash J_{u_{y}^{\xi}}\right)+\mathcal{H}^{0}\left(J_{u_{y}^{\xi}}\right)\right) d \mathcal{H}^{n-1}(y) \leq \\
\leq & \int_{\Omega^{\xi}}\left(\mathcal{L}^{1}\left(\Omega_{y}^{\xi}\right)+\int_{\Omega_{y}^{\xi} \backslash J_{u_{y}^{\xi}}}\left|\nabla\left(u_{y}^{\xi}\right)\right|^{2} d t+\mathcal{H}^{0}\left(J_{u_{y}^{\xi}}\right)\right) d \mathcal{H}^{n-1}(y) \leq \\
\leq & \int_{\Omega^{\xi}} c\left[1+\liminf _{r \rightarrow+\infty} \int_{\Omega_{y}^{\xi}}\left(\left(v_{r}\right)_{y}^{\xi}\left|\nabla\left(\left(u_{r}\right)_{y}^{\xi}\right)\right|^{2}+\kappa\left(\frac{\psi\left(v_{r}\right)}{\varepsilon_{r}}+a \varepsilon_{r}^{p-1}\left|\nabla\left(v_{r}\right)\right|^{p}\right)\right) d t\right],
\end{aligned}
$$

where $c:=\operatorname{diam}(\Omega)+1+\alpha$ and we have used (4.11)-(4.14). The last term in the previous estimate is bounded by (4.11) and this gives $u \in G S B D(\Omega)$.

Now we integrate on $\Omega^{\xi}$ both sides of (4.13); by (4.10)-(4.12), (2.8), and the Fubini Theorem we find (4.8) as $\kappa \rightarrow 0$.

Now we observe that

$$
\int_{\Omega}(e(u) \xi \cdot \xi-w)^{2} d x \leq \liminf _{k \rightarrow+\infty} \int_{\Omega} v_{k}\left(e\left(u_{k}\right) \xi \cdot \xi-w\right)^{2} d x
$$

follows from (4.8) for every $w \in L^{2}(\Omega)$. Indeed, (4.15) trivially holds if $w$ is piecewise constant on a Lipschitz partition of $\Omega$; then a density argument proves (4.15) for an arbitrary $w \in L^{2}(\Omega)$.

The next step is to deduce by (4.15) that

$$
e\left(u_{k}\right) v_{k}^{\frac{1}{2}} \rightarrow e(u) \text { weakly in } L^{2}\left(\Omega, \mathbb{M}_{s y m}^{n \times n}\right) .
$$

To this aim, we first extract a subsequence $\left(u_{l}, v_{l}\right)$ of $\left(u_{k}, v_{k}\right)$ such that $v_{l} \rightarrow 1 \mathcal{L}^{n}$-a.e. in $\Omega$ and $e\left(u_{l}\right) v_{l}^{\frac{1}{2}} \rightarrow A$ weakly in $L^{2}\left(\Omega, \mathbb{M}_{s y m}^{n \times n}\right)$, for a suitable $A \in L^{2}\left(\Omega, \mathbb{M}_{\text {sym }}^{n \times n}\right)$. Now we apply (4.15) to $w=A \xi \cdot \xi-t z$, for $t \in \mathbb{R}$ and $z \in L^{2}(\Omega)$. After an easy computation we find

$$
\int_{\Omega}((e(u)-A) \xi \cdot \xi)^{2} d x+2 t \int_{\Omega} z(e(u)-A) \xi \cdot \xi d x \leq \liminf _{l \rightarrow+\infty} \int_{\Omega} v_{l}\left(\left(e\left(u_{l}\right)-A\right) \xi \cdot \xi\right)^{2} d x .
$$

As $t \rightarrow \pm \infty$, the previous inequality leads to a contradiction unless $\int_{\Omega} z(e(u)-A) \xi \cdot \xi d x=0$ for every $z \in L^{2}(\Omega)$ and every $\xi \in \mathbb{R}^{n}$, namely unless $e(u)=A \mathcal{L}^{n}$-a.e. in $\Omega$. Therefore (4.16) holds true.

We use now the Egorov Theorem to find, in correspondence of $\mu>0$, a Borel set $B_{\mu} \subset \Omega$ such that $\mathcal{L}^{n}\left(\Omega \backslash B_{\mu}\right)<\mu$ and $v_{k}>1-\mu$ on $B_{\mu}$ for $k$ large. An easy computation then shows that

$$
e\left(u_{k}\right) \chi_{B_{\mu}} \rightarrow e(u) \chi_{B_{\mu}} \text { weakly in } L^{2}\left(\Omega, \mathbb{M}_{s y m}^{n \times n}\right) .
$$

We are now in a position to apply [11, Theorem 2.3.1], so that

$$
\int_{B_{\mu}} Q(e(u)) \leq \liminf _{k \rightarrow+\infty} \int_{\Omega} Q\left(v_{k}, e\left(u_{k}\right) \chi_{B_{\mu}}\right) d x \leq \int_{\Omega} Q\left(v_{k}, e\left(u_{k}\right)\right) d x .
$$


By the absolute continuity of the Lebesgue integral the left-hand side of the previous inequality tends to $\int_{\Omega} Q(e(u)) d x$ as $\mu \rightarrow 0$, and this concludes the proof of (4.6).

Proof of (4.7). For this part we refer to [18, Proposition 1]. We only point out that arguing again by slicing, using (2.6) and the coarea formula, we find

$$
\alpha \int_{J_{u}^{\xi}}\left|\nu_{u} \cdot \xi\right| d \mathcal{H}^{n-1} \leq \liminf _{k \rightarrow+\infty} \int_{\Omega}\left(\frac{\psi\left(v_{k}\right)}{\varepsilon_{k}}+a \varepsilon_{k}^{p-1}\left|\nabla v_{k}\right|^{p}\right) d x,
$$

namely the set $J_{u}^{\xi}$ replaces the set $J_{u}$ appearing in [18, Inequality (58)]. Nevertheless, inequality (4.18) still holds true with $J_{u}$ in place of $J_{u}^{\xi}$ by (2.9), being the set

$$
\left\{\xi \in \mathbb{S}^{n-1}: \mathcal{H}^{n-1}\left(J_{u} \backslash J_{u}^{\xi}\right)=0\right\}
$$

dense in $\mathbb{S}^{n-1}$. Eventually, inequality (4.7) follows from this and from a classical localization argument.

Let us prove now the upper estimate. We denote by $F_{2}^{\prime \prime}$ the $\Gamma$-limsup of $F_{k}$ in $L^{2}\left(\Omega, \mathbb{R}^{n}\right) \times L^{1}(\Omega)$.

Theorem 4.4. Assume (a)-(g) and assume that $\Omega$ has Lipschitz boundary. Then

$$
F_{2}^{\prime \prime}(u, 1) \leq \Phi(u),
$$

for every $u \in G S B D^{2}(\Omega) \cap L^{2}\left(\Omega, \mathbb{R}^{n}\right)$.

Proof. The crucial point of this proof is the approximation of a function $u \in G S B D^{2}(\Omega) \cap L^{2}\left(\Omega, \mathbb{R}^{n}\right)$ with more regular functions, through the Density Theorem 3.1. Precisely, it provides a sequence $u_{k} \in S B V^{2}\left(\Omega, \mathbb{R}^{n}\right) \cap L^{\infty}\left(\Omega, \mathbb{R}^{n}\right)$ such that

$$
u_{k} \rightarrow u \text { in } L^{2}\left(\Omega, \mathbb{R}^{n}\right) \quad \text { and } \quad \Phi\left(u_{k}\right) \rightarrow \Phi(u),
$$

so that if we prove that $u_{k}$ satisfies (4.19), then also $u$ satisfies (4.19), being $F_{2}^{\prime \prime}$ lower semicontinuous in $L^{2}\left(\Omega, \mathbb{R}^{n}\right) \times L^{1}(\Omega)$.

The proof of (4.20) for functions in $S B V^{2}\left(\Omega, \mathbb{R}^{n}\right) \cap L^{\infty}\left(\Omega, \mathbb{R}^{n}\right)$ is now standard (see, for instance, $[12,13])$. Let us give a brief description of the construction of the recovery sequence, following the approach of [22, Theorem 3.3].

Using a local reflection argument we reduce to prove the statement for $\Omega$ open cube in $\mathbb{R}^{n}$. Now Theorem 3.2 and Remark 3.3 allow us to assume in addition that $\overline{J_{u}}$ is contained in $\Omega$ and that $u$ satisfies properties (1)-(3) of Theorem 3.2. Moreover, it is not restrictive to consider only the case when $\overline{J_{u}}$ is a $(n-1)$-simplex, which we denote by $S$.

Let us fix a sequence of constants $\sigma_{k}$ such that $\eta_{k} / \sigma_{k} \rightarrow 0$ and $\sigma_{k} / \varepsilon_{k} \rightarrow 0$. We introduce now the sets $A_{k}, A_{k}^{\prime}, B_{k}$, and $B_{k}^{\prime}$, defined precisely in [22, Theorem 3.3]. Here we just recall that $A_{k} \cup A_{k}^{\prime}$ is a neighborhood of $S$ such that

$$
\mathcal{L}^{n}\left(A_{k}\right) \leq c \sigma_{k} \quad \text { and } \quad \mathcal{L}^{n}\left(A_{k}^{\prime}\right) \leq c \sigma_{k}^{2}
$$

and the set $B_{k} \cup B_{k}^{\prime}$ is a layer which envelops $A_{k} \cup A_{k}^{\prime}$ and satisfies

$$
\mathcal{L}^{n}\left(B_{k}\right) \leq c \varepsilon_{k} \quad \text { and } \quad \mathcal{L}^{n}\left(B_{k}^{\prime}\right) \leq c \varepsilon_{k}^{2},
$$

for a suitable constant $c<+\infty$.

Also the definition of the recovery sequence $\left(u_{k}, v_{k}\right)$ is given in analogy with [22, Theorem 3.3]. In particular $u_{k}$ is set equal to $u$ out of $A_{k} \cup A_{k}^{\prime}$ and it is a linear link in $A_{k}$ in the direction of $e_{n}$. With this definition $u_{k}$ is a Lipschitz function in $\Omega \backslash A_{k}^{\prime}$ with constant $c / \sigma_{k}$, where $c<+\infty$. To check this it is sufficient to apply the arguments given in [18, Theorem 3.3, Inequalities (71)-(78)] to each components $u_{k}^{i}$ of $u_{k}$. Thanks to the Mc Shane Theorem we are now able to define $u_{k}$ also in $A_{k}^{\prime}$ in a way that

$$
\left|D u_{k}\right| \leq c / \sigma_{k} \mathcal{L}^{n} \text {-a.e. in } \Omega \text {. }
$$

In addition, we define $v_{k}$ by $\eta_{k}$ in $A_{k} \cup A_{k}^{\prime}$, by 1 out of $A_{k} \cup A_{k}^{\prime} \cup B_{k} \cup B_{k}^{\prime}$, and in a way that, in terms of energy, the transition in $B_{k} \cup B_{k}^{\prime}$ is optimal. 
As for the computation of $F_{k}\left(u_{k}, v_{k}\right)$, we only observe that

$$
\int_{A_{k} \cup A_{k}^{\prime}} Q\left(\eta_{k}, e\left(u_{k}\right)\right) d x \rightarrow 0,
$$

by $(4.21),(4.23)$, and by the convergence $\eta_{k} / \sigma_{k} \rightarrow 0$. This concludes the proof, since the computation for the other terms work as in [22, Theorem 3.3].

Let us prove the $\Gamma$-convergence Theorem 4.1 for $\left(G_{k}\right)$.

Proof of Theorem 4.1. Let us introduce $H: L^{1}\left(\Omega, \mathbb{R}^{n}\right) \times L^{1}(\Omega) \rightarrow[0,+\infty]$, defined by

$$
H(u, v):= \begin{cases}\int_{\Omega}|u-g|^{2} d x & \text { if } u \in L^{2}\left(\Omega, \mathbb{R}^{n}\right), \\ +\infty & \text { otherwise. }\end{cases}
$$

On the one hand we notice that

$$
F^{\prime}+H \leq G^{\prime}
$$

where $F^{\prime}, G^{\prime}$ represent the $\Gamma$-lower limits of $F_{k}$ and $G_{k}$ in $L^{1}\left(\Omega, \mathbb{R}^{n}\right) \times L^{1}(\Omega)$ and we have used the fact that $H$ is lower semicontinuous in $L^{1}\left(\Omega, \mathbb{R}^{n}\right) \times L^{1}(\Omega)$. Then if $(u, v) \in L^{1}\left(\Omega, \mathbb{R}^{n}\right) \times L^{1}(\Omega)$ satisfies $G^{\prime}(u, v)<+\infty$, one deduces by Theorem 4.3 that $u$ belongs to $G S B D^{2}(\Omega) \cap L^{2}\left(\Omega, \mathbb{R}^{n}\right)$, $v=1 \mathcal{L}^{n}$-a.e., and

$$
\Psi(u)=\Phi(u)+H(u, 1) \leq G^{\prime}(u, 1) .
$$

On the other hand if $u \in G S B D^{2}(\Omega) \cap L^{2}\left(\Omega, \mathbb{R}^{n}\right)$, then the continuity of $H$ in $L^{2}\left(\Omega, \mathbb{R}^{n}\right) \times L^{1}(\Omega)$ and Theorem 4.4 yield

$$
G^{\prime \prime}(u, 1) \leq G_{2}^{\prime \prime}(u, 1)=F_{2}^{\prime \prime}(u, 1)+H(u, 1) \leq \Phi(u)+H(u, 1)=\Psi(u),
$$

where $G^{\prime \prime}, G_{2}^{\prime \prime}$ represent the $\Gamma$-upper limits of $G_{k}$ in $L^{1}\left(\Omega, \mathbb{R}^{n}\right) \times L^{1}(\Omega)$ and in $L^{2}\left(\Omega, \mathbb{R}^{n}\right) \times L^{1}(\Omega)$. The thesis follows from (4.26) and (4.27).

A key point for the proof of Corollary 4.2 is the compactness of a minimizing sequence. This is obtained in the following proposition, through a characterization which relates compactness of sequences to compactness of slices (see [1, Theorem 6.6] and [17, Theorem 10.7]).

Proposition 4.5. Let $\left(u_{k}, v_{k}\right) \in L^{1}\left(\Omega, \mathbb{R}^{n}\right) \times L^{1}(\Omega)$ be such that $\left(G_{k}\left(u_{k}, v_{k}\right)\right)$ is bounded. Then $v_{j} \rightarrow 1$ in $L^{1}(\Omega)$ and a subsequence $\left(u_{j}\right)$ of $\left(u_{k}\right)$ converges in $L^{1}\left(\Omega, \mathbb{R}^{n}\right)$ to a function $u \in L^{2}\left(\Omega, \mathbb{R}^{n}\right)$.

Proof. The proof follows the lines of [17, Theorem 11.1]. It is sufficient to prove the statement for any open set which is relatively compact in $\Omega$. Furthermore we assume that $\Omega$ is a finite union of open rectangles and we extend each function by zero out of $\Omega$. Let $M<+\infty$ be such that $G_{k}\left(u_{k}, v_{k}\right) \leq M$.

Since $\left(F_{k}\left(u_{k}, v_{k}\right)\right)$ is bounded, the sequence $v_{k}$ converges to 1 in $L^{1}(\Omega)$ and $\mathcal{L}^{n}$-a.e. in $\Omega$, up to subsequences. We fix now $k \in \mathbb{N}$ and $\xi \in \mathbb{S}^{n-1}$. For $y \in \Omega^{\xi}$ we introduce the one-dimensional functional $F_{y, k}: L^{1}\left(\Omega_{y}^{\xi}\right) \times L^{1}\left(\Omega_{y}^{\xi}\right) \rightarrow \mathbb{R}$ defined by

$$
F_{y, k}(w, z):= \begin{cases}\int_{\Omega_{y}^{\xi}}\left(z|\nabla w|^{2}+\frac{\psi(z)}{\varepsilon_{k}}+a \varepsilon_{k}^{p-1}|\nabla(z)|^{p}\right) d t & \text { if }(w, z) \in H^{1}\left(\Omega_{y}^{\xi}\right) \times V_{y, \eta_{k}} \\ +\infty & \text { otherwise }\end{cases}
$$

where $V_{y, \eta_{k}}:=\left\{z \in W^{1, p}\left(\Omega_{y}^{\xi}\right): \eta_{k} \leq z \leq 1 \mathcal{H}^{1}\right.$-a.e. in $\left.\Omega_{y}^{\xi}\right\}$. We also define for $\lambda>0$

$$
\begin{gathered}
\hat{A}_{k}^{\xi, \lambda}:=\left\{y \in \Omega^{\xi}:\left(u_{k}\right)_{y}^{\xi} \in H^{1}\left(\Omega_{y}^{\xi}\right), F_{y, k}\left(\left(u_{k}\right)_{y}^{\xi},\left(v_{k}\right)_{y}^{\xi}\right) \leq \lambda\right\}, \quad \hat{B}_{k}^{\xi, \lambda}:=\Omega^{\xi} \backslash \hat{A}_{k}^{\xi, \lambda}, \\
A_{k}^{\xi, \lambda}:=\left\{x \in \Omega: \Pi^{\xi}(x) \in \hat{A}_{k}^{\xi, \lambda}\right\}, \quad B_{k}^{\xi, \lambda}:=\left\{x \in \Omega: \Pi^{\xi}(x) \in \hat{B}_{k}^{\xi, \lambda}\right\},
\end{gathered}
$$

being $\Pi^{\xi}(x)$ the projection of $x$ on the plane $\Pi^{\xi}$. Since $\left(F_{k}\left(u_{k}, v_{k}\right)\right)$ is bounded, the Chebychev Inequality and the Fubini Theorem yield

$$
\mathcal{L}^{n}\left(B_{k}^{\xi, \lambda}\right) \leq \operatorname{diam}(\Omega) \frac{c}{\lambda}
$$


Here and henceforth $c$ represents a finite constant; in particular $c(\delta)$ will denote its possible dependence on $\delta$. For $\mu>0$ and $t \in \mathbb{R}$, we introduce the truncation function $\tau_{\mu}(t):=-\mu \vee t \wedge \mu$ and we set

Let

$$
w_{k, \mu}^{\xi, \lambda}:= \begin{cases}\tau_{\mu}\left(u_{k} \cdot \xi\right) & \text { in } A_{k}^{\xi, \lambda} \\ 0 & \text { in } B_{k}^{\xi, \lambda}\end{cases}
$$

$$
\phi(t):=\int_{0}^{t} \psi^{\frac{1}{q}} d s \quad \text { for } t \in[0,1]
$$

and let $\tilde{c}$ be a constant which uniformly bounds $\phi\left(v_{k}\right)$. For $\delta>0$ we are able to find $\lambda_{\delta}$ and $\mu_{\delta}$ large enough to guarantee

$$
\tilde{c}|| u_{k} \cdot \xi-w_{k, \mu_{\delta}}^{\xi, \lambda_{\delta}} \|_{L^{1}\left(\mathbb{R}^{n}\right)}<\delta
$$

uniformly with respect to $k$. Indeed, let $\mu_{\delta}>0$ be such that $s \leq \frac{\delta}{4 M} s^{2}$ for $s \geq \mu_{\delta}$ and let $\lambda_{\delta}$ be such that $\mu_{\delta} \mathcal{L}^{n}\left(B_{k}^{\xi, \lambda_{\delta}}\right) \leq \delta / 2$, (this is possible by (4.28)). Therefore we find

$$
\begin{aligned}
\int_{\Omega}\left|u \cdot \xi-w_{k, \mu_{\delta}}^{\xi, \lambda_{\delta}}\right| d x & =\int_{\left\{|u \cdot \xi|>\mu_{\delta}\right\}}\left|u \cdot \xi-w_{k, \mu_{\delta}}^{\xi, \lambda_{\delta}}\right| d x+\int_{B_{k}^{\xi, \lambda} \cap \cap\left\{|u \cdot \xi| \leq \mu_{\delta}\right\}}|u \cdot \xi| d x \\
& \leq 2 \int_{\left\{|u \cdot \xi|>\mu_{\delta}\right\}}|u| d x+\mu_{\delta} \mathcal{L}^{n}\left(B_{k}^{\xi, \lambda_{\delta}}\right) \\
& \leq \frac{\delta}{2 M} \int_{\Omega}|u|^{2} d x+\frac{\delta}{2}=\delta .
\end{aligned}
$$

For simplicity in what follows we write $w_{k}$ in place of $w_{k, \mu_{\delta}}^{\xi, \lambda_{\delta}}$.

In order to apply [17, Lemma 10.7], we set

$$
U:=\left(\phi\left(v_{k}\right) u_{k}\right), \quad V_{\delta}^{\xi}:=\left(\phi\left(v_{k}\right) w_{k}\right),
$$

and we show that for every $k$ and for $\mathcal{H}^{n-1}$-a.e. $y \in \Omega^{\xi}$ we have

$$
\int_{\mathbb{R}}\left|\left(\phi\left(v_{k}\right) w_{k}\right)_{y}^{\xi}(t+h)-\left(\phi\left(v_{k}\right) w_{k}\right)_{y}^{\xi}(t)\right| d t \leq \omega_{\delta}(h) \quad \text { for } h \in(0,1),
$$

for a suitable modulus of continuity $\omega_{\delta}$ independent on $k, y$, and $\xi$. To this aim we check that for every $k$ and for $\mathcal{H}^{n-1}$-a.e. $y \in \Omega^{\xi}$ the function $\left(\phi\left(v_{k}\right) w_{k}\right)_{y}^{\xi}$ satisfies all requirements of [17, Lemma 10.8], uniformly with respect to $k$ and $y$.

First note that for every $k$ and for $\mathcal{H}^{n-1}$-a.e. $y \in \Omega^{\xi}$ the function $\left(\phi\left(v_{k}\right) w_{k}\right)_{y}^{\xi}$ belongs to $S B V^{2}(\mathbb{R}) \cap$ $L^{\infty}(\mathbb{R})$, that $\mathcal{H}^{0}\left(\left(J_{w_{k}}\right)_{y}^{\xi}\right) \leq c$, and that $\left\|\left(\phi\left(v_{k}\right) w_{k}\right)_{y}^{\xi}\right\|_{L^{\infty}(\mathbb{R})} \leq c(\delta)$. Moreover the Young Inequality, the estimate $\phi(t) \leq c t$, and the Hölder Inequality yield

$$
\begin{gathered}
\int_{\Omega_{y}^{\xi}}\left|\nabla\left(\left(\phi\left(v_{k}\right) w_{k}\right)_{y}^{\xi}\right)\right| d t \leq \\
c(\delta) \int_{\Omega_{y}^{\xi}}\left(\frac{\psi\left(\left(v_{k}\right)_{y}^{\xi}\right)}{\varepsilon_{k}}+\varepsilon_{k}^{p-1}\left|\nabla\left(\left(v_{k}\right)_{y}^{\xi}\right)\right|^{p}\right) d t+c(\operatorname{diam}(\Omega))^{\frac{1}{2}}\left(\int_{\Omega_{y}^{\xi}}\left(v_{k}\right)_{y}^{\xi}\left|\nabla\left(w_{k}\right)_{y}^{\xi}\right|^{2} d t\right)^{\frac{1}{2}} \leq c(\delta) .
\end{gathered}
$$

We are now in a position to apply [17, Lemma 10.8], so that $(4.30)$ holds with $\omega_{\delta}(h):=c(\delta) h$. Through [17, Lemma 10.7], inequalities (4.29) and (4.30) imply the existence of a subsequence $\left(\phi\left(v_{j}\right) u_{j}\right)$ of $\left(\phi\left(v_{k}\right) u_{k}\right)$ and of a function $\tilde{u} \in L^{1}\left(\Omega, \mathbb{R}^{n}\right)$ such that $\phi\left(v_{j}\right) u_{j} \rightarrow \tilde{u}$ in $L^{1}\left(\Omega, \mathbb{R}^{n}\right)$. The Fatou Lemma also gives $\tilde{u} \in L^{2}\left(\Omega, \mathbb{R}^{n}\right)$. Eventually the thesis follows for $u:=\tilde{u} / \phi(1)$.

We conclude proving Corollary 4.2 .

Proof of Corollary 4.2. Let us fix $k$ and check that the functional $G_{k}$ achieves its infimum. If $\left(u_{j}, v_{j}\right)$ is a minimizing sequence for $G_{k}$, the sequence $\left(u_{j}\right)$ belongs to $H^{1}\left(\Omega, \mathbb{R}^{n}\right)$, is bounded in $L^{2}\left(\Omega, \mathbb{R}^{n}\right)$, and the sequence of symmetric gradients $e\left(u_{j}\right)$ is bounded in $L^{2}\left(\Omega, \mathbb{M}_{\text {sym }}^{n \times n}\right)$. By Korn's inequality this implies that $\left(u_{j}\right)$ is bounded in $H^{1}\left(\Omega, \mathbb{R}^{n}\right)$, so that there exist a subsequence of $\left(u_{j}\right)$, not relabelled, and a function $u \in H^{1}\left(\Omega, \mathbb{R}^{n}\right)$ such that $u_{j} \rightarrow u$ weakly in $H^{1}\left(\Omega, \mathbb{R}^{n}\right)$. 
Being $\left(v_{j}\right)$ bounded in $W^{1, p}(\Omega)$ we also infer that there exists a further subsequence of $\left(v_{j}\right)$, not relabelled, and a function $v \in V_{\eta_{k}}$ such that

$$
v_{j} \rightarrow v \text { weakly in } W^{1, p}(\Omega) \text { and } \mathcal{L}^{n} \text {-a.e. in } \Omega \text {. }
$$

By the Ioffe-Olech semicontinuity theorem (see, for instance, [11, Theorem 2.3.1.]) and the Fatou lemma we deduce that

$$
\int_{\Omega} Q(v, e(u)) d x \leq \liminf _{j \rightarrow+\infty} \int_{\Omega} Q\left(v_{j}, e\left(u_{j}\right)\right) d x \quad \text { and } \quad \int_{\Omega}|u-g|^{2} d x \leq \liminf _{j \rightarrow+\infty} \int_{\Omega}\left|u_{j}-g\right|^{2} d x
$$

hold, therefore $(u, v)$ minimizes $G_{k}$.

Now a sequence $\left(u_{k}, v_{k}\right)$ of minimizers of $G_{k}$ is compact in $L^{1}\left(\Omega, \mathbb{R}^{n}\right) \times L^{1}(\Omega)$ by Proposition 4.5. Let $(u, 1)$ be the limit point of a subsequence, not relabelled, of $\left(u_{k}, v_{k}\right)$. By Theorem 4.1 and by a general result of $\Gamma$-convergence, we infer that $(u, 1)$ is a minimizer for $G$ and that the convergence of minimum values holds.

To conclude the proof it remains to show that $u_{k} \rightarrow u$ in $L^{2}\left(\Omega, \mathbb{R}^{n}\right)$. To this aim it is sufficient to prove that

$$
\int_{\Omega}\left|u_{k}-g\right|^{2} d x \rightarrow \int_{\Omega}|u-g|^{2} d x
$$

By the convergence of the minimum values $G_{k}\left(u_{k}, v_{k}\right) \rightarrow G(u, v)$, the following inequalities

$$
\Phi(u) \leq \liminf _{k \rightarrow+\infty} F_{k}\left(u_{k}, v_{k}\right) \quad \text { and } \quad \int_{\Omega}|u-g|^{2} d x \leq \liminf _{k \rightarrow+\infty} \int_{\Omega}\left|u_{k}-g\right|^{2} d x
$$

(holding true by Theorem 4.3 and the lower semicontinuity of $H$ ) are actually equalities. This gives (4.32) and concludes the proof.

\section{ACKNOWLEDGEMENTS}

This material is based on work supported by the ERC Advanced Grant n. 290888 "QuaDynEvoPro". The author would like to acknowledge Prof. Gianni Dal Maso for many interesting discussions.

\section{REFERENCES}

[1] G. Alberti, G. Bouchitté, P. Seppecher, Phase transition with the line-tension effect, Arch. Rational Mech. Anal. 144 (1998), 1-46.

[2] L. Ambrosio, A. Coscia, G. Dal Maso, Fine properties of functions with bounded deformation, Arch. Rational Mech. Anal. 139 (1997), 201-238.

[3] L. Ambrosio, N. Fusco, and D. Pallara, "Functions of Bounded Variation and Free Discontinuity Problems", The Clarendon Press, Oxford University Press, New York, 2000.

[4] L. Ambrosio, V. M. Tortorelli, Approximation of functionals depending on jumps by elliptic functionals via T-convergence, Comm. Pure Appl. Math. 43 (1990), 999-1036.

[5] L. Ambrosio, V. M. Tortorelli, On the approximation of free discontinuity problems, Boll. Un. Mat. Ital. B (7) 6 (1992), 105-123.

[6] G. Bellettini, A. Coscia, G. Dal Maso, Special Functions of Bounded Deformation, Preprint SISSA, Trieste, 1995.

[7] G. Bellettini, A. Coscia, G. Dal Maso, Compactness and lower semicontinuity properties in $\operatorname{SBD}(\Omega)$, Math. Z. 228 (1998), 337-351.

[8] B. Bourdin, Numerical implementation of the variational formulation for quasi-static brittle fracture, Interfaces Free Bound. 9 (2007), 411-430.

[9] B. Bourdin, G. A. Francfort, J.-J. Marigo, Numerical experiments in revisited brittle fracture, J. Mech. Phys. Solids 48 (2000), 797-826.

[10] B. Bourdin, G. A. Francfort, J.-J. Marigo, The variational approach to fracture, J. Elasticity 91 (2008), 5-148.

[11] G. Buttazzo, "Semicontinuity, Relaxation and Integral Representation in the Calculus of Variation", Pitman Research Notes in Mathematics Series 203, Longman Scientific \& Technical, Harlow, 1989.

[12] A. Chambolle, An approximation result for special functions with bounded deformation, J. Math. Pures Appl. (9) 83 (2004), 929-954.

[13] A. Chambolle, Addendum to: "An approximation result for special functions with bounded deformation" (J. Math. Pures Appl. (9) 83 (2004), no. 7, 929-954; MR2074682], J. Math. Pures Appl. (9) 84 (2005), 137-145.

[14] G. Cortesani, Strong approximation of GSBV functions by piecewise smooth functions, Ann. Univ. Ferrara Sez. VII (N.S.) 43 (1997), 27-49.

[15] G. Cortesani, R. Toader, A density result in SBV with respect to non-isotropic energies, Nonlinear Anal. 38 (1999), 585-604. 
[16] G. Dal Maso, "An Introduction to $\Gamma$-Convergence", Progress in Nonlinear Differential Equations and their Applications, 8, Birkhäuser Boston Inc., Boston, MA, 1993.

[17] G. Dal Maso, Generalised functions of bounded deformation, J. Eur. Math. Soc. (JEMS), to appear. Preprint available on: http://cvgmt.sns.it/paper/1704/.

[18] G. Dal Maso, F. Iurlano, Fracture models as $\Gamma$-limits of damage models, Commun. Pure Appl. Anal. 12 (2013), 1657-1686. Available on: http://www.aimsciences.org/journals/displayArticlesnew.jsp?paperID=7924.

[19] H. Federer, "Geometric measure theory", Die Grundlehren der mathematischen Wissenschaften, Band 153, Springer-Verlag New York Inc., New York, 1969.

[20] G. A. Francfort, J.-J. Marigo, Revisiting brittle fracture as an energy minimization problem, J. Mech. Phys. Solids 46 (1998), 1319-1342.

[21] G. A. Francfort, J.-J. Marigo, Cracks in fracture mechanics: a time indexed family of energy minimizers, "Variations of domain and free-boundary problems in solid mechanics (Paris, 1997)", 197-202, Solid Mech. Appl. 66, Kluwer Acad. Publ., Dordrecht, 1999.

[22] F. Iurlano, Fracture and plastic models as $\Gamma$-limits of damage models under different regimes, Adv. Calc. Var., to appear. Available on: http://www.degruyter.com/view/j/acv.ahead-of-print/acv-2011-0011/ acv-2011-0011.xml.

[23] R. Temam, "Problèmes mathématiques en plasticité", Méthodes Mathématiques de l'Informatique [Mathematical Methods of Information Science] 12, Gauthier-Villars, Montrouge, 1983.

[24] R. Temam, G. Strang, Functions of bounded deformation, Arch. Rational Mech. Anal. 75 (1980/81), 7-21.

(Flaviana Iurlano) SISSA, Via Bonomea 265, 34136 Trieste, Italy

E-mail address: iurlano@sissa.it 